Eidg. Technische Hochschule, Zürich --Fortbildungskurs der EAWAG, 1964

\title{
Grundlagen \\ für die Dimensionierung der Belüftung bei Belebtschlammanlagen
}

Von K. WUHRMANN, EAWAG, Zürich 


\section{Einleitung}

Das Problem der Berechnung der Beluiftungsintensität bei einer Belebtschlammanlage ist im Prinzip äusserst einfach und übersichtlich. Es sind zwei Grössen zu kennen, nämlich:

- der Sauerstoffverbrauch des Belebtschlammgemisches bei der Reinigung des zufliessenden Abwassers (O-Bedarf) und

- die Sauerstofflösungsleistung der vorgesehenen Belüftungsapparatur bei den gegebenen Verhältnissen bezüglich Abwasserqualität und herrschenden physikalischen Bedingungen (Deckung des O-Bedarfs).

Die Sauerstoffeintragsleistung eines bestimmten Belüftungssystems ist ausschliesslich eine Frage chemisch-physikalischer Parameter und hat mit den Abbauvorgängen in einer Belebtschlammanlage direkt nichts zu tun. Wir werden uns deshalb mit der Deckung des O-Bedarfs eines Belebtschlammsystems nicht beschäftigen, da uns hier in erster Linie die Ermittlung des O-Bedarfs als unmittelbar von den Fermentationsvorgängen bestimmte Grösse interessiert.

Grundsätzlich muss die Belüftungseinrichtung die Sauerstoffmenge $W_{\mathrm{o}}$ eintragen, die zur Deckung des Bedarfs $q_{g}$ notwendig ist, und ferner muss sie jenen Mehrbetrag liefern können, der zur Aufrechterhaltung einer gewünschten Sauerstoff konzentration von $\mathrm{c} \mathrm{mg} \mathrm{O} / \mathrm{l}$ im Belüftungsbecken $\left(0<c<c_{s}\right)$ benötigt wird.

Normalerweise wird der Wert $W_{\mathrm{o}}$, bzw. $k_{t}$ unter Standardbedingungen experimentell ermittelt und kann für eine bestimmte Belüftungseinrichtung angegeben werden. Die verlangte Eintragsleistung ins Belüftungsbecken ist nun aber nicht $W_{\text {o }}$, sondern $W_{c}$, da ja eine gewisse Sauerstoffkonzentration $c$ im Belebtschlammgemisch stets aufrechterhalten bleiben soll. Es ist 


$$
W_{c}=W_{o} \frac{c_{s}}{c_{s}-c} .
$$

Da wir verlangen, dass $W_{o}=q_{g}$ sein soll, wird also

$$
W_{c}=q_{g} \frac{c_{s}}{c_{s}-c} \quad\left[\mathrm{~g} \mathrm{O} / \mathrm{m}^{3}{ }_{B B} \cdot \mathrm{h}\right]
$$

und gleichzeitig ist

$$
W_{c}=k_{t}\left(c_{s}-c\right) \quad\left[\mathrm{g} \mathrm{O} / \mathrm{m}^{3}{ }_{B B} \cdot \mathrm{h}\right],
$$

so dass die Belüftungskonstante $k_{t}$, die eine bestimmende Masszahl für ein Belüftungssystem ist, errechnet werden kann:

$$
k_{t}=q_{g} \frac{c_{s}}{\left(c_{s}-c\right)^{2}} \quad\left[\mathrm{~h}^{-1}\right] \text {. }
$$

\section{Benötigte Sauerstoffkonzentrationen im Belüftungsbecken}

Damit ein aerobes Belebtschlammsystem einen optimalen Wirkungsgrad aufweisen kann (im Sinne grösster Substanzelimination pro Zeiteinheit und GewichtseinheitSchlamm) muss im Milieu u. a. eine Sauerstoffspannung vorhanden sein, die es gestattet, um jede einzelne Zelle der Schlammflocken mindestens die kritische Sauerstoffspannung stets aufrechtzuerhalten (unbegrenzte Menge aller übrigen Stoffwechselsubstrate vorausgesetzt). Die kritische O-Spannung liegt vermutlich bei allen Bakterien eines Belebtschlamms in der Grössenordnung von $\mathrm{I}-2 \mathrm{~mm} \mathrm{Hg}$, entsprechend rund o, I $\mathrm{mg} \mathrm{O} / \mathrm{l}$ bei Belebtschlammtemperaturen um $15^{\circ} \mathrm{C}$ und $760 \mathrm{~mm}$ Druck. Wir haben früher angegeben (WUHRMANN [18, 20]), wie sich die Flockenbildung bezüglich der Sauerstoffversorgung der Einzelzellen auswirkt. Für mittlere Verhältnisse bezüglich O-Verbrauch der Einzelzelle, Diffusionskoeffizient von Sauerstoff im Flockenmaterial usw. ergibt sich ein Zusammenhang gemäss Abb. I. Aus der Darstellung entnimmt man sofort, dass für Flockengrössen, wie sie in gut turbulenten Belüftungsbecken vermutet werden dürfen (Durchmesser der kompakten Zellhaufen nicht grösser als $0,5 \mathrm{~mm}$ ), eine ausreichende $\mathrm{O}-\mathrm{V}$ ersorgung der Zellen im Flockenzentrum (mindestens O,I mg O/l um diese Zellen) bei einer Konzentration von etwa $2-3 \mathrm{mg} \mathrm{O} / 1$ im Belebtschlammgemisch gewährleistet ist. Diese Zahl ist etwas höher als sie VIEHL [IS] in seinen systematischen Versuchen gefunden hat (allerdings bei sehr kleinen Schlammbelastungen und dementsprechend geringen Atmungsgeschwindigkeiten des Schlamms). Sie stimmt mit den praktischen Erfahrungen bei mittel- bis hochbelasteten Anlagen überein und deckt sich mit den Beobachtungen unseres Faktorenversuchs (WuHRMANN [2I]).

1) Liste der Bezeichnungen am Schluss des Aufsatzes. 


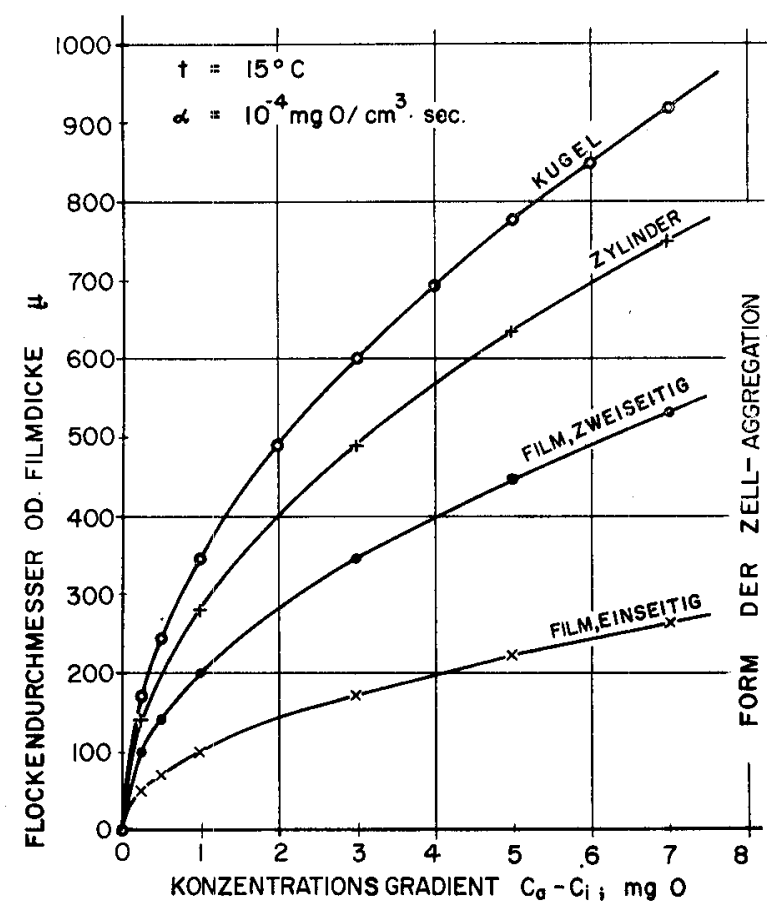

Abbildung 1

Sauerstoffversorgung von Zellen im Innern von Belebtschlammflocken oder Tropfkörperfilmen. Abszisse: Konzentrationsgradient von Sauerstoff von der Flockenoberfläche bis zur innersten Zelle. Ordinate: Dicke der Zellschicht. Beispiel: Sauerstoffkonzentration im Abwasser an der Flockenoberfläche $3 \mathrm{mg} / \mathrm{l}$, gewünschte Sauerstoffkonzentration um die innersten Zellen $0,1 \mathrm{mg} / \mathrm{l}$. Maximaler Flockendurchmesser (kugelige Form) etwa $600 \mu$, (zylindrische Form) etwa $480 \mu$. Schichtdicke ebener Flocken (beidseitig zugänglich) etwa $340 \mu$, (einseitig zugänglich) etwa $170 \mu$. (Aus WuHrmann [18])

Zusammenfassend kommen wir zur Schlussfolgerung, dass für die nachfolgend diskutierte Berechnung der Belïftungsintensität eine Basiszabl für die Sauerstoffkonzentration c im Belüftungsbecken von 2 bis böchstens $3 \mathrm{mg}$ O/l anzunehmen ist.

\section{Begriffsbestimmung für endogene und Substratatmung}

Wenn eine kausal begründete Berechnung des Sauerstoffbedarfs in einer Belebtschlammanlage gegeben werden soll, sind einige physiologische und chemische Gegebenheiten zu berücksichtigen. Der Sauerstoffverbrauch eines Belebtschlamm-Abwassergemischs setzt sich aus zwei grundverschiedenen Komponenten zusammen: 
a) Sauerstoffverbrauch für die rein chemische Oxydation von Verbindungen, die bei den gegebenen $\mathrm{pH}-\mathrm{Verhältnissen}$ und den vorhandenen Sauerstoffspannungen während der Belüftungsdauer in eine andere Oxydationsstufe übergehen können (z.B. Oxydation von $\mathrm{Fe}^{2+} \rightarrow \mathrm{Fe}^{3+}$. Der entsprechende O-Verbrauch ist stöchiometrisch leicht zu errechnen oder experimentell zu bestimmen. Er spielt bei vorwiegend städtischem Abwasser eine vernachlässigbar kleine Rolle.

b) Sauerstoffverbrauch des Energiestoffwechsels der Schlammorganismen.

Die in der neueren Abwasserliteratur zu findenden Rezepte für die Berechnung dieses Sauerstoffbedarfs gehen meistens davon aus, dass er die Summe aus der endogenen Atmung $q_{e}$ des Belebtschlamms und einem Betrag für die Substratatmung $q_{s}$, d.h. die Veratmung der Schmutzstoffe (z.B. gemessen als $\mathrm{BSB}_{5}$ ) darstelle:

$$
q_{g}=q_{e}+q_{S} \quad\left[\mathrm{~g} \mathrm{O} / \mathrm{m}_{B B}^{3} \cdot \mathrm{h}\right] .
$$

Dieses Vorgehen kann allerdings nur dann zu annehmbaren Ergebnissen führen, wenn die beiden Begriffe «endogene» und "Substratatmung» korrekt angewendet und ihnen die richtigen Masszahlen gegeben werden.

Die endogene Atmung lässt sich exakt definieren. Sie entspricht der Atmung der Zellen, denen ausschliesslich zelleigene Oxydationssubstrate für den Energiestoffwechsel zur Verfügung stehen. Die endogene Atmung kann deshalb nur an Zellen gemessen werden, die in einem vollständig substratfreien Milieu aufgeschwemmt sind. Für Belebtschlamm heisst das, dass man die Schlämme in anorganischen Pufferlösungen waschen muss und erst dann die Atmungsmessung vornehmen darf. Die endogene Atmung ist keine konstante Grösse für eine gegebene Organismenpopulation. Sie ändert sich je nach dem physiologischen Zustand der Organismen. Zellen, die unmittelbar vor der Respirationsmessung aus einem substratreichen Milieu abgetrennt wurden und dementsprechend reichliche Mengen von respirierbaren Reservestoffen enthalten, besitzen eine höhere endogene Atmung, als wenn sie vollständig verarmt sind. Ferner hängt die Grösse der endogenen Atmung, bezogen auf Zelltrockengewicht, Zellstickstoff oder dgl., bei einem Belebtschlamm in entscheidendem Masse davon ab, welcher Prozentsatz der Gesamttrockensubstanz in Form von lebenden Zellen vorliegt. Wir werden auf diesen Punkt in einem späteren Abschnitt nochmals zurückkommen.

Die Angaben über endogene Respiration von Belebtschlämmen in der Abwasserliteratur sind leider oft unbrauchbar, weil entscheidende Parameter, wie beispielsweise die Messtemperatur oder die Vorbereitung des 
Schlamms für die Messung (Waschen in Pufferlösung) nicht angegeben werden. Häufig sind auch ungenügende Messeinrichtungen verwendet worden (üblicher Fehler: ungenügende Turbulenz des Schlamms zur vollständigen und dauernden Einstellung eines Gleichgewichts zwischen Sauerstoff konsumation und Sauerstoffzufuhr bei jeder einzelnen Zelle des Schlamms). Nach unserer Erfahrung ist die mikromanometrische Methode nach wie vor das technisch einfachste, schnellste und genaueste Verfahren für solche Untersuchungen. Schon WOOLDRIDGE und STANDFAST [I6] stellten fest, dass bei korrekter Technik der Sauerstoffverbrauch eines Belebtschlamms innert weiter Grenzen erwartungsgemäss als streng proportional zur Schlammkonzentration gefunden wird. Wir haben (WUHRMANN [I7]) mit Untersuchungen über die Atmungsgeschwindigkeit in Funktion der Sauerstoffspannung im Reaktionsgefäss und der Konzentration des Belebtschlamms gezeigt, dass bei Einwaagen von 2-3 mg Trockensubstanz $/ \mathrm{ml}$ und Luft in der Gasphase eines Mikromanometers bei den üblichen Schüttelfrequenzen die volle Atmungsgeschwindigkeit gewährleistet ist.

Die Substratatmung ist ein umstrittener und jedenfalls nicht genau definierbarer Begriff. Es ist eine Beobachtungstatsache, dass die Atmung einer Zelle stark über das endogene Niveau ansteigt im Moment, wo ihr ein Energiesubstrat im Aussenmilieu zur Verfügung gestellt wird. Die Atmungs-

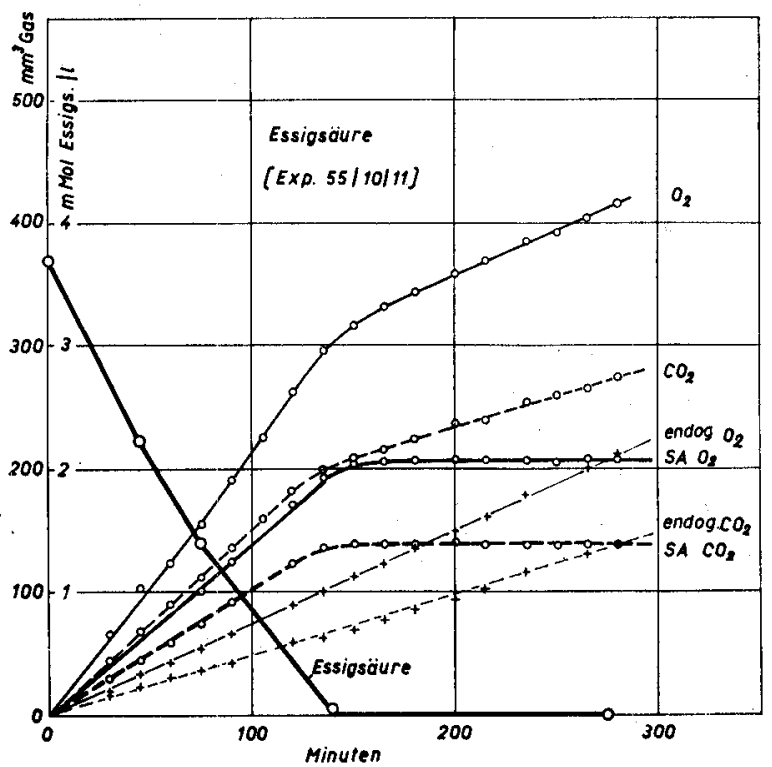

Abbildung $2 a$ 
steigerung hält so lange an, bis das exogene Substrat erschöpft ist und fällt dann auf das endogene Niveau zurück (vorausgesetzt, dass kein Organismenzuwachs in der Beobachtungsperiode erfolgt ist). Abb. $2 a$ u. $b$ geben zwei Beispiele für die Atmungsreaktionen von Belebtschlämmen nach Zusatz einer Reinsubstanz. Diese graphischen Versuchsprotokolle enthalten alle Atmungswerte und auch die Konzentrationsveränderung der zugesetzten Atmungssubstrate. Die sog. «Substratatmung» (SA) ist als Differenz des Sauerstoff konsums für die endogene Atmung und die Atmung mit Substrat berechnet und ebenfalls eingezeichnet worden.

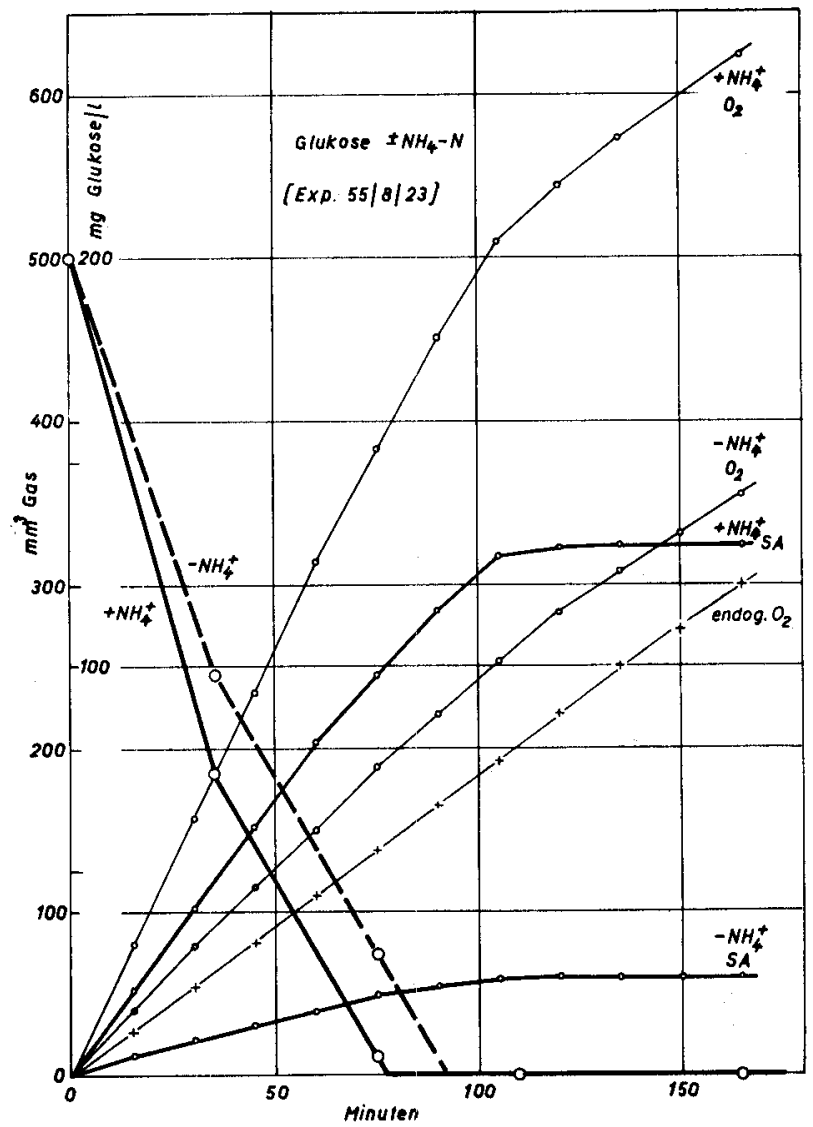

Abbildung $2 b$

Sauerstoffaufnahme und $\mathrm{CO}_{2}$-Abgabe von Belebtschlämmen bei endogener Atmung und bei der Respiration eines Substrats. Mikromanometrische Messungen. Temperatur bei allen Versuchen $25^{\circ} \mathrm{C}$. Barcroftmanometer. $a$ Essigsäure als Substrat, $b$ Glukose $\pm \mathrm{NH}_{4}^{+}$als Substrat (Aus Wuhrmann und von Beust [17]). 
Wir haben vorstehend rein phänomenologisch die Substratatmung als additive Grösse behandelt. $\mathrm{Ob}$ dies physiologisch korrekt ist, kann nicht eindeutig gesagt werden. Man findet, dass bei den einzelnen Bakterienarten offensichtlich keine einheitliche Reaktionsweise besteht. So fanden Clifton und Sobek $[3,4]$ bei Bacillus cereus, dass die endogene Atmung bei Anwesenheit von Glukose im Aussenmilieu um 20 bis $40 \%$ zurückgedrängt wurde ( "teilweise additive Substratatmung»). RAMSEY [I2] konnte dagegen bei Staphylococcus aureus zeigen, dass die endogene Atmung durch die gleichzeitige Veratmung exogen gebotener Glukose überhaupt nicht beeinflusst wird ( «streng additive Substratatmung»). Bei diesem Bakterium dienen Aminosäuren im Aminosäure-Pool der Zelle uiberwiegend als Substrat für die endogene Atmung. - Trotz dieser Uneinheitlichkeit der Reaktionsweise bei den Bakterien (wobei Unabhängigkeit von endogener und Substratrespiration überwiegend vorkommen dürfte) betrachten wir es als zulässig, für praktische Zwecke die endogene und die Substratatmung voneinander zu trennen, nicht zuletzt auch deshalb, weil wir dann die experimentellen Beobachtungen leichter in Berechnungswerte für die Belüftung umsetzen können.

Gewisse Schwierigkeiten bei der Bestimmung der Grösse der Substratatmung liegen darin, dass sie für ein und dieselbe Substanz ganz verschiedene Werte annehmen kann. Gemäss dem Stoffwechselschema in Abb. 3 ist ja die Proportion zwischen der Substratmenge, welche in den Baustoffwechsel und der Menge, die in den Betriebsstoffwechsel eingeht, für den absoluten Wert der Substratatmung (gemessen als additive Grösse zur endogenen Atmung) massgebend.

Zufluss

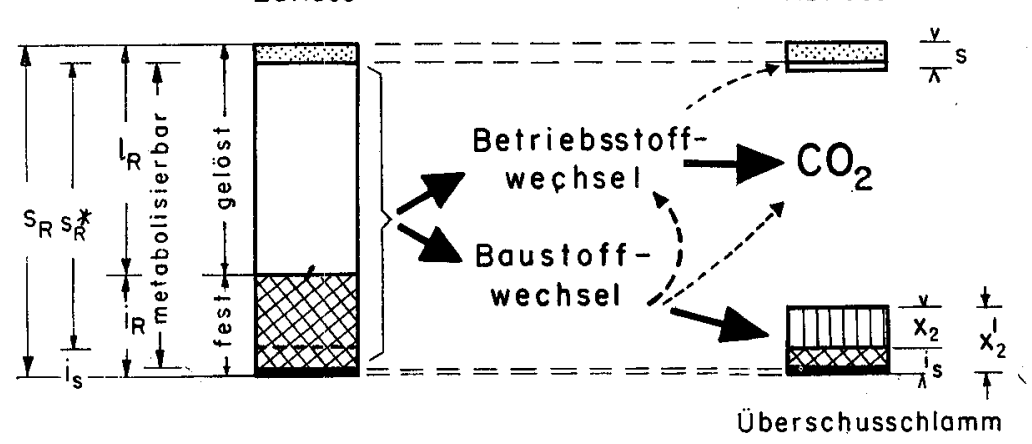

Abbildung 3

Schema der Bilanz des organischen Kohlenstoffs innerhalb eines Belebtschlammsystems. (AUS WUHRMAN N [19]) 
Als Beispiel sei auf Abb. $2 b$ verwiesen. Bei diesem Versuch zeigte ein Belebtschlamm für die gleiche Menge Glukose als Substrat eine «Substratatmung» von $3,26 \mathrm{~mol} \mathrm{O} / \mathrm{mol}$ Glukose $(=54,4 \%$ der Theorie für vollständige Oxydation) bei Anwesenheit von $\mathrm{NH}_{4}^{+}$, dagegen $0,67 \mathrm{~mol} \mathrm{O} / \mathrm{mol}$ Glukose (=II, $2 \%$ der Theorie für vollständige Oxydation) bei Abwesenheit von $\mathrm{NH}_{4}^{+}$.

Diese unterschiedliche Benützung eines Substrats für den Bau- oder den Energiestoffwechsel, je nach Anwesenheit anderer Substrate oder in Abhängigkeit von den weiteren Milieubedingungen, ist schon lange bekannt (BARKER [I]; CLIFTON [2]) und der Nachweis dieser Reaktionsweise bei einer Mischbiozönose wie dem Belebtschlamm bietet an sich nichts Neues. Es ist aber die Schlussfolgerung zu ziehen, dass der $B S B_{5}$ des Abwassers ein ganz ungeeignetes Mass für die Abschät $=$ ung des Sauerstoffbedarfs in einer Anlage darstellt, da aller Voraussicht nach die Proportion Bau-/Energie-Stoffwechsel in der $\mathrm{BSB}_{5}$-Flasche wesentlich anders sein wird als im Belüftungsbecken, ganz abgesehen davon, dass der $\mathrm{BSB}_{5}$ bestenfalls ein qualitatives, aber niemals ein quantitatives Mass für die Substratkonzentration darstellen kann.

\section{Grösse der endogenen Atmung von Belebtschlamm}

Da der absoluten Grösse der endogenen Atmung bei der Berechnung des Sauerstoffbedarfs einer Belebtschlammanlage erhebliche praktische Bedeutung zukommt, müssen wir ihre Beziehungen zu den übrigen Betriebsparametern eines Belebtschlammsystems genauer prüfen. In einer Untersuchung, die in ähnlicher Weise angelegt war wie unser Faktorenversuch, haben ORFord, HeUKeLEKIAN und IsENBERG [II] ebenfalls die endogene Atmung des Belebtschlamms gemessen und die Ergebnisse in Funktion der Schlammbelastung (mit BSB ${ }_{5}$ ) korreliert. Andere systematische Beobachtungen in dieser Richtung liegen bisher nicht vor, wenn auch von zahlreichen Autoren Angaben über die endogene Atmung von Belebtschlamm veröffentlicht worden sind. Wir geben zunächst die Ergebnisse bekannt, die wir bei der regelmässigen Messung der endogenen Atmung der Belebtschlämme im Faktorenversuch (WUHRMANN [2I]) gewinnen konnten. Bei jedem Einzelversuch wurde innerhalb der Analysenperiode an 3 Tagen die Atmung des gewaschenen, in Pufferlösung suspendierten Schlamms mikromanometrisch bestimmt (anfänglich mit Barcroft-, später mit WarburgManometern; jede Messung erfolgte im Doppel). Gleichzeitig wurde auch die Substratatmung nach Zusatz von doppelt konzentriertem Abwasser gemessen. Die letztern Resultate werden wir später noch erwähnen. Die Be- 
stimmungen erfolgten bei $25^{\circ} \mathrm{C}$. Da der Temperaturkoeffizient der Schlammatmung zwischen 25 und $0^{\circ} \mathrm{C}$ ziemlich genau $\Theta_{10}=2$, o beträgt (WUHRMANN [I7]), können die Beobachtungswerte ohne weiteres $\mathrm{für}$ eine andere (tiefere) Temperatur umgerechnet werden. Das Rechnungsverfahren ist am Schluss dieses Abschnitts in Erinnerung gerufen.

Die in jedem Versuch gefundenen Werte (pro Versuch 3 Messtage, jede Bestimmung im Doppel, d.h. $3 \times 2=6$ Bestimmungen pro Versuch, d.h. pro Schlammtyp) in den Sommer- und Winterperioden sind als $Q_{O e}$ (in $\mathrm{mg}$ $\mathrm{O} / \mathrm{g}$ Trockensubstanz $\cdot \mathrm{h}$ ) in Tab. I zusammengefasst ${ }^{2}$ ). Wie wir bereits in der früheren Arbeit über den Faktorenversuch betonten, ist nur eine statistische Betrachtung dieser Aufstellung zulässig, da den Einzelwerten ein zu geringes Gewicht zukommt (keine Wiederholung der Einzelversuche!).

Aus der Varianzanalyse des gesamten Versuchsmaterials ( 54 Experimente) in Tab. 2 ergibt sich sofort, dass nur die Temperatur und die Schlammkonzentration im Beluiftungsbecken einen erkennbaren Einfluss auf $Q_{O e}$ ausüben, alle andern Faktoren sind nicht wirksam und ferner bestehen auch keine gegenseitigen Abhängigkeiten zwischen den untersuchten Variablen.

Das Gesamtergebnis der statistischen Untersuchung lässt sich wie folgt zusammenfassen:Bei gegebenerAbwasserzusammensetzung und-konzentration vermindert sich die Grösse der endogenen Atmung des Belebtschlamms innerhalb des beobachteten Bereiches von Betriebsbedingungen ungef ähr proportional zur'Zunahme derSchlammkonzentration im Belïftungsbecken.

Die Ziffern in den Tab. I-3 sagen nun allerdings noch nichts aus über den gesuchten Zusammenhang zwischen Schlammzustand und Grösse der endogenen Atmung. Es scheint deshalb zweckmässig, beispielsweise dieSchlammbelastung oder das Schlammalter mit der Grösse der endogenen Atmung zu korrelieren.

Scblammbelastung und endogene Atmung. Die Werte für die Schlammbelastungen $\left(\mathrm{kg} \mathrm{BSB} B_{5} / \mathrm{kg}\right.$ TS · Tag) für jedes Einzelexperiment des Faktorenversuchs sind in der früheren Arbeit tabelliert. Aus der vorstehenden Varianzanalyse geht hervor, dass ein Einfluss der Sauerstoffspannung im Belüftungsbecken auf die endogene Atmung nicht nachzuweisen ist. Wir haben deshalb die Messungen der je drei Versuche mit gleichem O-Gehalt zu neuen Mittelwerten zusammengefasst. Zusammen mit einigen weiteren

\footnotetext{
2) Wir diskutieren im folgenden in der Regel nur die Werte für die endogene Atmung, gemessen an der Schlammtrockensubstanz. In gleicher Weise kann man die Atmungswerte aber auch beziehen auf den organischen Anteil des Schlamms, seinen Gehalt an organischem Stickstoff oder Kohlenstoff usw. Da diese anderen Masszahlen nichts grundsätzlich Neues bringen, verzichten wir im allgemeinen auf ihre Wiedergabe.
} 
Tabelle 1

Endogene Atmung von Belebtschlamm in Abhängigkeit von hydraulischer Raumbelastung, Schlammkonzentration und Sauerstoffgehalt im Belüftungsbecken. Atmungsmessung bei $25^{\circ} \mathrm{C}$. Schlämme $2 \times$ in Pufferlösung $p H 7,5$ gewaschen. Angaben als $\mathrm{mg}$ O/g Schlammtrockensubstanz und Stunde

\begin{tabular}{|c|c|c|c|c|c|c|c|}
\hline \multirow[t]{2}{*}{$\begin{array}{l}\mathrm{O}_{2}-\text { Konz. } \\
\mathrm{BB} \mathrm{mg} / \mathrm{l}\end{array}$} & \multirow[t]{2}{*}{$\begin{array}{l}\text { Schlamm- } \\
\text { Konz. BB } \\
\text { mg/l }\end{array}$} & \multicolumn{3}{|c|}{$\begin{array}{l}\text { Mittl. Temp. BB }=>13^{\circ} \mathrm{C} \\
\text { Hydr. Raumbelastung } \\
\mathrm{m}^{3} / \mathrm{m}^{3} \text {. Tag }\end{array}$} & \multicolumn{3}{|c|}{$\begin{array}{l}\text { Mitt1. Temp. BB }=<11^{\circ} \mathrm{C} \\
\text { Hydr. Raumbelastung } \\
\mathrm{m}^{3} / \mathrm{m}^{3} \text {. Tag }\end{array}$} \\
\hline & & 58 & 29 & 9 & 58 & 29 & 9 \\
\hline 1 & $\begin{array}{r}600 \\
3300 \\
6000\end{array}$ & $\begin{array}{l}17,1 \\
16,6 \\
13,9\end{array}$ & $\begin{array}{r}17,2 \\
16,5 \\
8,7\end{array}$ & $\begin{array}{r}17,9 \\
11,3 \\
9,4\end{array}$ & $\begin{array}{l}15,7 \\
14,7 \\
11,9\end{array}$ & $\begin{array}{l}16,3 \\
16,7 \\
11,0\end{array}$ & $\begin{array}{r}18,5 \\
16,9 \\
9,7\end{array}$ \\
\hline 4 & $\begin{array}{r}600 \\
3300 \\
6000\end{array}$ & $\begin{array}{l}15,7 \\
11,4 \\
12,6\end{array}$ & $\begin{array}{r}16,2 \\
15,1 \\
9,6\end{array}$ & $\begin{array}{c}(21,7) \\
10,1 \\
7,4\end{array}$ & $\begin{array}{l}18,0 \\
16,3 \\
11,1\end{array}$ & $\begin{array}{l}21,2 \\
16,0 \\
12,2\end{array}$ & $\begin{array}{l}18,6 \\
14,3 \\
12,6\end{array}$ \\
\hline 7 & $\begin{array}{r}600 \\
3300 \\
6000\end{array}$ & $\begin{array}{l}14,9 \\
13,3 \\
11,7\end{array}$ & $\begin{array}{r}19,5 \\
14,4 \\
7,7\end{array}$ & $\begin{array}{r}12,1 \\
8,6 \\
11,3\end{array}$ & $\begin{array}{l}22,6 \\
18,0 \\
14,3\end{array}$ & $\begin{array}{l}21,7 \\
15,3 \\
12,6\end{array}$ & $\begin{array}{l}20,3 \\
16,7 \\
13,2\end{array}$ \\
\hline
\end{tabular}

Tabelle 2

Varianzanalyse der Werte für die endogene Atmung Qoe der Belebtschlämme aus allen Winter-und Sommermonaten (je 27 Experimente)

\begin{tabular}{lrrrrl}
\hline Variabilität & $F_{\mathrm{g}}$ & \multicolumn{1}{c}{$S Q$} & \multicolumn{1}{c}{$D Q$} & $f$ & $P$ \\
\hline Temperatur $t$ & 1 & 77,04 & 77,04 & 17,6 & $<0,001$ \\
$R_{h}$ & 2 & 12,43 & 6,22 & 1,42 & $\geqslant 0,1$ \\
$x_{1}$ & 2 & 429,20 & 214,60 & 94 & $<0,001$ \\
$O$ & 2 & 2,46 & $\mathbf{1}, 23$ & 0,28 & $\gg 0,1$ \\
$t \times R_{h}$ & 2 & 7,73 & 3,86 & 0,88 & $\gg 0,1$ \\
$t \times x_{1}$ & 2 & 8,62 & 4,31 & 0,98 & $\geqslant 0,1$ \\
$t \times O$ & 2 & 41,04 & 10,52 & 2,40 & $>0,1$ \\
Rest & 40 & $\mathbf{1 7 5 , 4 6}$ & 4,38 & & \\
Insgesamt & 53 & 746,25 & - & & \\
\end{tabular}

Tabelle 3

Durchschnitswerte für die endogene. Atmung $Q_{0 \text { e }}$ bei $25^{\circ} \mathrm{C}$ in mg O/g TS $\cdot h$, gemittelt über die Raumbelastungen 9 bis $58 \mathrm{~m}^{3} / \mathrm{m}^{3}$. Tag und die O-Konzentrationen von 1 bis $7 \mathrm{mg} / \mathrm{l}$

\begin{tabular}{rlll}
\hline$x_{1}$ & $<11^{\circ} \mathrm{C}$ & $>13^{\circ} \mathrm{C}$ & So/Wi \\
\hline 600 & 19,2 & 16,9 & 0,88 \\
3300 & 16,1 & 13,0 & 0,87 \\
6000 & 12,0 & 10,2 & 0,85 \\
\hline
\end{tabular}

Vertrauensgrenze für $P=0,1: \pm 1,28 \mathrm{mg} \mathrm{O} / \mathrm{g} \mathrm{TS} \cdot \mathrm{h}$. 
Beobachtungen, die unabhängig vom Faktorenversuch, aber unter vergleichbaren Bedingungen gewonnen wurden, ergibt sich dann ein ziemlich guter funktioneller Zusammenhang z.mischen endogener Atmung des Schlamms und der Schlammbelastung gemäss Abb. 4. Sowohl der Einfluss der Temperatur als auch der Durchlaufszeit im Belüftungsbecken kommen dabei deutlich zum Ausdruck. Man stellt vor allem fest, dass bei Schlammbelastungen von $S_{b}<$ etwa I, $5 \mathrm{~kg} \mathrm{BSB} / \mathrm{kgTS} \cdot$ Tag die endogene Atmung stark von der Schlammbelastung abhängig wird. Bei grösseren Belastungen (bis zu den untersuchten Maximalwerten von $\mathrm{rd}$. $10 \mathrm{~kg} \mathrm{BSB} \mathrm{B}_{5} / \mathrm{kg}$ TS $\cdot \mathrm{Tag}$ ) ergeben sich gleichbleibende Werte von $\mathrm{I} 6$ bis $18 \mathrm{~g} O / \mathrm{kg}$ TS $\cdot \mathrm{h}$ bei Temperaturen im Belüftungsbecken von $>\mathrm{I} 3^{\circ} \mathrm{C}$, bzw. I 8 bis $20 \mathrm{~g} \mathrm{O} / \mathrm{kg}$ TS $\cdot \mathrm{h}$ bei Belüftungstemperaturen $<\mathrm{II}^{\circ} \mathrm{C}$. Benützt man die Schlammbelastung mit organischem Kohlenstoff als Bezugsgrösse, so ergibt sich ein analoges Bild wie in Abb.4, so dass wir auf seine Wiedergabe verzichten können.

In der bereits erwähnten Untersuchung von ORFORD et al. [II], in welcher Schlämme aus dem Belastungsbereich von etwa o,os bis etwa $2 \mathrm{~kg} \mathrm{BSB}_{5} / \mathrm{kg}$ TS ·Tag geprift wurden, stellten die Autoren einen gesicherten linearen

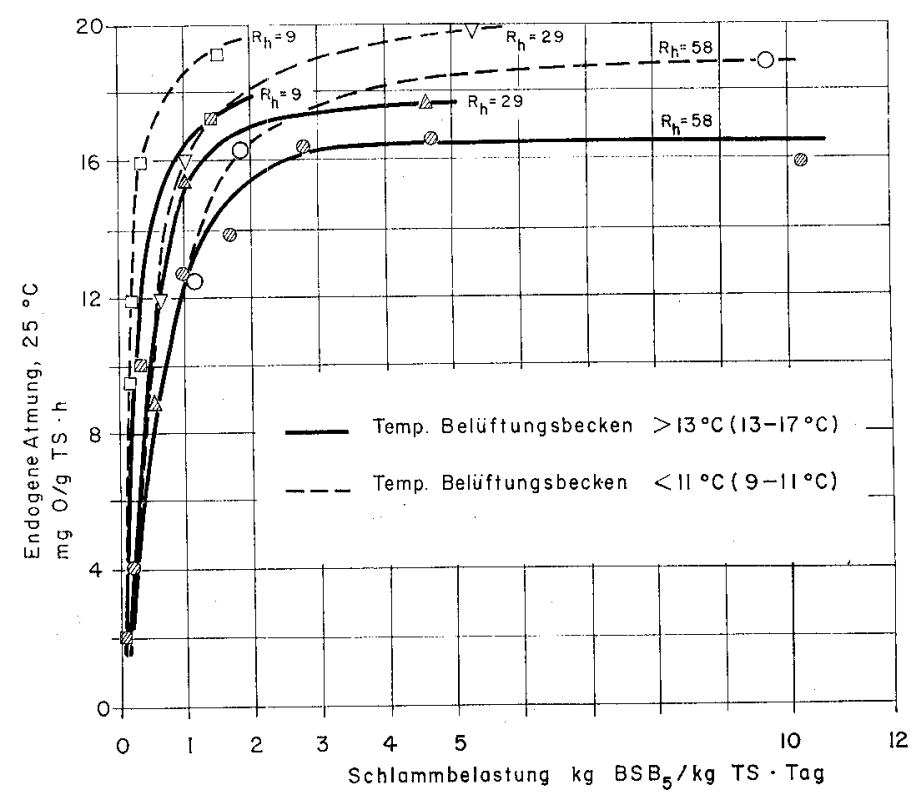

Abbildung 4

Endogene Atmung $Q_{O e}$ (bei $25^{\circ} \mathrm{C}$ ) der Belebtschlämme des Faktorenversuchs.(WUHRmann [21])

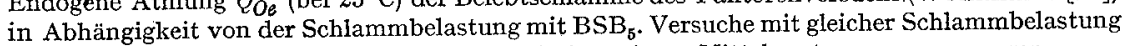
bei verschiedener Sauerstoffspannung sind zu einem Mittelwert zusammengezogen. 
Zusammenhang zwischen endogener A tmung und dem log der Schlammbelastung fest. Die Streuungen ihrer Versuche sind allerdings sehr gross. Trägt man unsere Beobachtungen ebenfalls in Funktion des log der Schlammbelastung auf, so findet man, dass die Kurve mit der besten Angleichung an die Messpunkte S-förmigen Charakter besitzt mit asymptotischen Annäherungen der Atmungswerte an $Q_{O e}\left(25^{\circ} \mathrm{C}\right)=$ etwa $1,5-2,5 \mathrm{mg}$ $\mathrm{O} / \mathrm{g}$ TS $\cdot \mathrm{h}$ bei $S_{b}<0,06$ und an $Q_{o}\left(25^{\circ} \mathrm{C}\right)=$ etwa $17 \mathrm{mg} \mathrm{O} / \mathrm{g}$ TS $\cdot \mathrm{h}$ bei $S_{b}>\mathrm{I}, 3 \mathrm{~kg} \mathrm{BSB} / \mathrm{kg}$ TS $\cdot$ Tag (Einfluss der hydraulischen Raumbelastung vernachlässigt).

Schlammalter und endogene Atmung. Als Schlammalter definieren wir die theoretische Aufenthaltszeit einer Schlammpartikel im Belebtschlammsystem (Berechnung: Quotient aus Gesamtüberschußschlamm-Ablass und Gesamtschlamminhalt des Systems, das heisst: Trockensubstanz im Belüftungsbecken + die Trockensubstanz im Nachklärbecken und in eventuellen Rückführsystemen dividiert durch die tägliche Trockensubstanz des Überschußschlamms + Schwebestoffe im Anlagenabfluss). Die entsprechenden Ziffern für den Faktorenversuch sind ebenfalls in der früheren Arbeit tabelliert. In Abb. 5 sind nun die Einzelpunkte aller Experimente zusammengefasst (eine Mittelbildung von Versuchsgruppen ist mit diesem Material wenig sinnvoll, da die Schlammalter in den Einzelexperimenten z.T. stark verschieden sind). Einige weitere Beobachtungen anlässlich anderer Versuche sind ebenfalls in Abb. 5 eingetragen. Angesichts der grossen Streuungen verzichten wir darauf, Kurvenzüge durch die Punkteschar zu legen. Die Darstellung zeigt aber mindestens qualitativ die generelle Tendenz einer Abnabme der endogenen Atmung mit zunebmendem Schlammalter. Die gemäss Abb. 4 auftretende «kritische» Schlammbelastung fällt bei unseren Versuchen mit einem Schlammalter von etwa 0,45 Tagen zusammen. Wir können also die Punkteschar so weit ordnen, dass im Gebiete von Schlammaltern unter 0,45 Tagen die endogene Atmung mehr oder weniger konstante Beträge annimmt. Ihr Rückgang mit zunehmendem Schlammalter beginnt erst von dieser Grenze an. Schlämme mit sehr hohem «Alter» (vgl. letzte Punkte, diese Messungen beziehen sich auf den Schlamm einer sogenannten Volloxydationsanlage, Überschußschlammproduktion = Schwebestoffe im Abfluss; nähere Beschreibung dieser Versuche bei HöRLER [8]) weisen nur mehr sehr kleine Atmungswerte auf (etwa $2 \mathrm{mg}$ $\mathrm{O} / \mathrm{g}$ TS $\cdot h$ bei $25^{\circ} \mathrm{C}$ ).

Es stellt sich nun die Frage, wie die Abb. 4 und $s$ zu interpretieren sind. Wie bereits angedeutet wurde, sind folgende zwei Ursachen kausal für die 


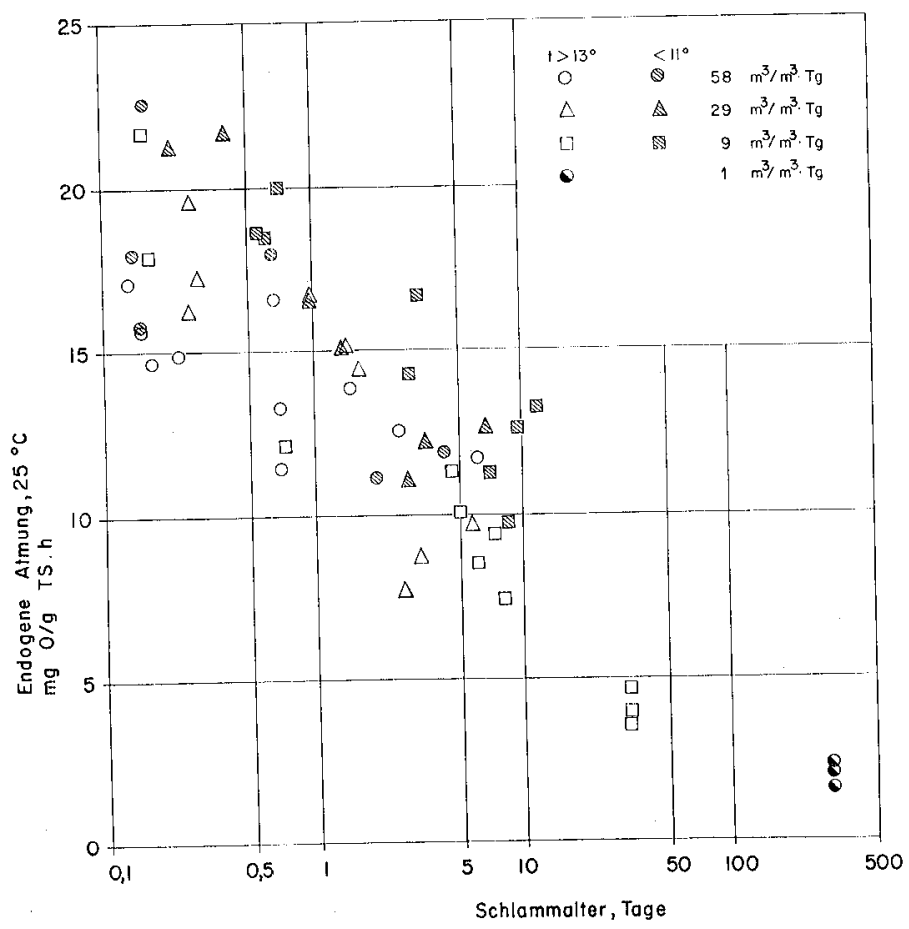

Abbildung 5

Endogene Atmung $Q_{O_{e}}$ (bei $25^{\circ} \mathrm{C}$ ) der Belebtschlämme des Faktorenversuchs in Abhängigkeit vom Schlammalter. Zusätzliche Beobachtungen aus Versuchen mit vergleichbaren Verhältnissen sind eingefügt für dic Schlammalter 40 und 300 Tage.

Änderung der endogenen Atmung in Funktion von Schlammbelastung oder Schlammalter zu diskutieren:

a) Verminderung der Zahl lebender Zellen pro Einheit Schlammtrockengewicht,

b) Verminderung der endogenen Atmungsintensität der einzelnen Zellen in Abhängigkeit vom Nährstoffangebot.

$\mathrm{Zu}$ a): RUCHOFFT, MCNAMEE und BUTTERFIELD [13], RUCHOFFT und SMITH [I4] sowie KeEFER und MEISEL [Io] haben gezeigt, dass die Elimination eines Substrats stark vom Schlammalter abhängig ist. Ferner konnten wir beweisen (WUHRMANN [17]), dass die Bakterien in einem Belebtschlamm (unter konstanten ökologischen Bedingungen wie Temperatur, $\mathrm{pH}, \mathrm{O}-$ Angebot usw.) bei einem Überschuss an Substrat pro Zeiteinheit eine gleichbleibende Menge resorbieren und deshalb die Abbauleistung 


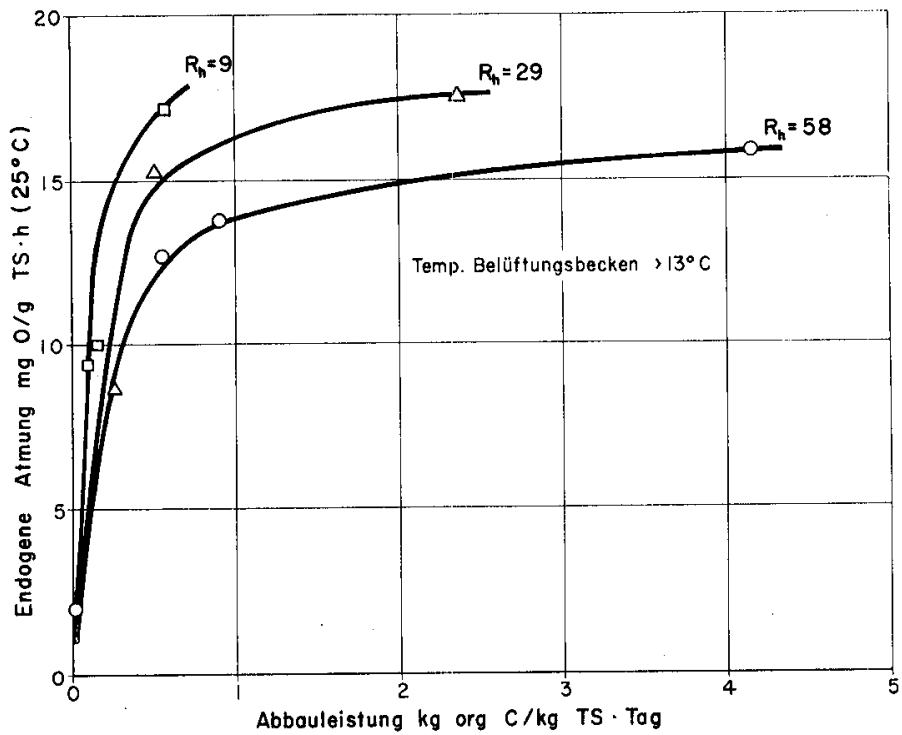

Abbildung 6

Abbauleistung von Belebtschlamm ( $\mathrm{kg}$ organischer $\mathrm{C} / \mathrm{kg}$ Trockensubstanz $\cdot$ Tag) in Funktion der endogenen Atmung. Mittelwerte von je drei Experimenten des Faktorenversuchs mit gleicher Raumbelastung und gleichem Schlammgehalt im Belüftungsbecken.

eines Schlamms pro Einheit Trockengewicht annähernd proportional zur Zahl der in ihr enthaltenen, lebenden Zellen sein muss. Dementsprechend muss sich auch die Respiration eines Schlamms, soweit sie nur von der Zahl der lebenden Zellen bestimmt ist, sich zur Geschwindigkeit der Substratelimination proportional verhalten. Die Beobachtungen in unserem Faktorenversuch gestatten nicht, einen derartigen quantitativen Rückschluss aus der Abbauleistung des Schlamms auf seinen Organismengehalt zu ziehen, da ja die Substratmenge nicht im Überschuss geboten wurde. Abb. 6 zeigt aber doch, dass die Abbauleistung, berechnet als Kilogramm Elimination von organischem $\mathrm{C} / \mathrm{kg}$ TS $\cdot$ Tag, stark ansteigt, wenn die endogene Atmung des Schlamms eine gewisse Grösse (etwa I5-I8 mg O/g TS $h$ h bei $25^{\circ} \mathrm{C}$ ) erreicht und überschreitet. Die Atmungsintensität nimmt von dieser Grenze an nicht mehr erkennbar zu.

Zu b): Aus den Versuchen von RAMSEY [12] geht eindeutig hervor, dass mit der Verarmung der Zellen an endogenen Respirationssubstraten (in seinen Untersuchungen: Aminosäure-Pool) die endogene Atmung von Bakterien stark zurückgeht. Diese Beobachtung lässt sich in beschränktem Umfange wohl auch auf den Belebtschlamm übertragen. Die begrenzte 
Substratmenge in einem Belebtschlammsystem genügt zwar kaum, um den Schlammorganismen die Anlage von wesentlichen endogenen Reserven von Atmungssubstraten zu ermöglichen. Die Auswirkung der «Verarmung» der einzelnen Zellen auf den Rückgang der endogenen Atmung dürfte deshalb klein sein.

Zusammenfassend kommen wir deshalb zur Schlussfolgerung, dass die beobacbteten Beziebungen zmischen endogener Atmung und Scblammalter, bzw. Scblammbelastung, im mesentlichen die Veränderungen der Zabl lebender Zellen im Scblamm in Funktion dieser beiden Parameter miderspiegeln.

Diese Feststellung gilt unseres Erachtens für alle Belebtschlämme aus Reinigungsanlagen, da in diesen Fermentationssystemen prinzipiell mit grossen Substratunterschüssen gearbeitet wird.

Um einen Einblick in die Grössenordnung der Proportion lebender Zellen am Gesamttrockengewicht eines Belebtschlamms zu erhalten, haben wir für den Faktorenversuch eine entsprechende Berechnung auf Grund der endogenen Atmung vorgenommen (Atmung, bezogen auf den Gehalt des Schlamms an organischem Stickstoff als Ausgangsbasis gewählt). Die höchste in einem Versuch beobachtete Schlammatmung ( $528 \mathrm{mg} \mathrm{O} / \mathrm{g} \mathrm{N} \cdot \mathrm{h}$ ) wurde willkürlich als einem Gehalt an lebenden Zellen von $90 \%$ zugehörig angenommen. Man findet dann die Durchschnittswerte gemäss Tab. 4 . Auch wenn es sich bei diesen Ziffern nur um rohe Schätzungen handelt, geben sie doch dem allgemeinen Trend Ausdruck, dass mit zunebmender Schlammkonzentration bei bescbränktem Substratangebot der Schlamm in einem Belebtscblammsystem stark an lebenden Zellen verarmt.

Für praktische $Z$ wecke dürften die Korrelationen von Abb. 4- 6 bei häuslichem Abwasser zur Bestimmung von $Q_{o e}$ ausreichend exakt sein. Es ist anzunehmen, dass mit stark zunehmender Abwasserkonzentration (aber ähnlicher chemischer Zusammensetzung wie das untersuchte häusliche Abwasser) keine ins Gewicht fallenden Veränderungen eintreten werden. Hingegen sind bei ganz andersartigen Abwässern, die zu Belebtschlämmen mit einer Organismenzusammensetzung führen, die von den untersuchten Schlämmen stark abweicht, eventuell andere Werte für $Q_{O e}$ zu erwarten. Es wäre deshalb eine interessante Aufgabe, die in der Literatur zerstreuten Angaben über die endogene Atmung von Belebtschlämmen zu sammeln und - soweit entsprechende Betriebsunterlagen vorhanden sind - sie mit den Korrelationen der Abb. 4, 5 und $6 \mathrm{zu}$ vergleichen. Dabei müssten allerdings die angewendeten Messverfahren kritisch beurteilt werden, denn speziell einige der publizierten « Makromethoden» dürften unrichtige (zu kleine) Resultate ergeben haben. Leider ist es auch nicht möglich, die Mess- 
Tabelle 4

Relativer Gewichtsanteil dev lebenden Zellen am Gesamttrockengewicht von Belebtschlamm bei verschiedenen Betriebsbedingungen. Berechnung aus endogener Atmung (mg O/g $N \cdot h)$. Basiswert: höchster Atmungswert von $528 \mathrm{mg} \mathrm{O} / \mathrm{g} \mathrm{N} \cdot \mathrm{h}=90 \%$

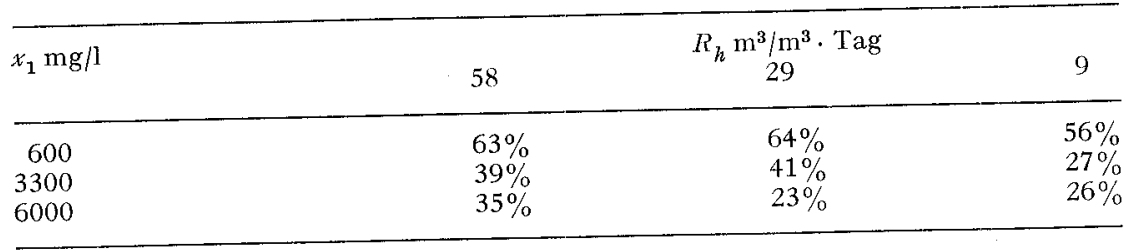

reihe, welche HUNKEN [9] veröffentlichte, genauer zu beurteilen, da die zu den untersuchten Schlämmen gehörigen Betriebsdaten der Belebtschlammanlagen nicht ersichtlich sind. Die von ihm hervorgehobenen, systematischen Unterschiede zwischen den Atmungsintensitäten der Schlämme aus der Anlage Stuttgart und Baden-Baden dürften wohl gerade ein Beispiel für die Wirksamkeit der Parameter Schlammbelastung oder Schlammalter abgeben.

Wir haben aus einigen unserer früheren Modellversuche über die Reinigung industrieller Abwässer ergänzendes Material herausgesucht und in Tab. 5 zusammengestellt:

Wie die Ziffern in 'Tab. 5 zeigen, ergeben diese Messungen keine neuen Grössenordnungen für die endogene Atmung bei Berücksichtigung der zugehörigen Betriebsdaten der Anlagen. Es ist dies auch nicht anders zu erwarten, da auch bei der Reinigung industrieller Abwässer (besonders zusammen mit häuslichem Abwasser) sich Mischbiozönosen ausbilden, deren Eigenschaften immer ungefähr ähnlich sind (Fälle mit ganz einseitig zusammengesetzten Abwässern vorbehalten).

Tabelle 5

Endogene Atmung von Belebtschlämmen aus Anlagen zur Reinigung von Industrieabwässern

\begin{tabular}{lllr}
\hline Abwasser & $\begin{array}{c}\text { Schlammbelastung } \\
\mathrm{kg} \mathrm{BSB} / \mathrm{kg} \text { TS·Tag }\end{array}$ & $\begin{array}{l}\text { Schlammalter } \\
\text { Tage }\end{array}$ & $\begin{array}{c}\text { Qoe }\left(25^{\circ} \mathrm{C}\right) \\
\mathrm{mg} \mathrm{O} / \mathrm{g} \mathrm{TS} \cdot \mathrm{h}\end{array}$ \\
\hline $\begin{array}{l}\text { Schlachthof } \\
\text { Schlachthof }\end{array}$ & 0,3 & & 9,9 \\
Gerberei + häusl. 1:1 & 0,73 & 3,5 & 20,7 \\
& 0,31 & 5,3 & 9,4 \\
häusl. + 40 mg/l & 0,18 & 11 & 5,1 \\
Kresole aus Industrie & 0,25 & 11 & 9,3 \\
Nescaféfabrik & 0,57 & 10 & 10,7 \\
\hline
\end{tabular}


Berecbnung des endogenen Anteils der Scblammatmung in einem Belüftungsbecken:

Da für ein und denselben Schlamm die endogene Atmung natürlich proportional zur Schlammkonzentration ist, ergibt sich ohne weiteres:

$$
\left.q_{e}=Q_{O e} \cdot x_{1} \quad\left[\mathrm{~g} \mathrm{O} / \mathrm{m}^{3}{ }_{B B} \cdot \mathrm{h}\right] \text { bei } t^{\circ} \mathrm{C}^{3}\right) \text {. }
$$

\section{Substratatmung}

Wir haben bereits darauf hingewiesen, dass die Grösse der Substratatmung ausschliesslich davon abhängt, welcher Anteil der von den Belebtschlammorganismen aus dem Abwasser aufgenommenen Substanzmenge in den Energiestoffwechsel abgef ührt wird. Für die Bestimmung der Substratatmung müssen deshalb zwei Grössen bekannt sein:

a) Die aus dem Abwasser in den Stoffwechsel einbezogenen Substratmengèn, vorteilhafterweise gemessen als organischer $\mathrm{C}$,

b) die davon in den Bau- und Reservestoffwechsel einerseits und unmittel-

bar in den Energiestoffwechsel andererseits übergef ührten Anteile.

Wir haben früher (WUHRMANN [2I] bereits dargestellt, dass man aus der Abbauleistung und dem produzierten Zellmaterial den biologischen Ausnützungsfaktor $Y$ berechnen kann, der nichts anderes darstellt als jenen Anteil verarbeiteten Substrats, der in den Bau- und Reservestoffwechsel einbezogen wurde. Es ist:

Gesamte, von den Organismen verarbeiteteSubstratmenge als organischer $C$ :

$$
\dot{C}_{\text {tot }}=\left(s_{R C}-s_{C}\right) \text {, }
$$

davon in Baustoffwechsel (= Zellmaterialproduktion):

$$
C_{B}=Y\left(s_{R C}-s_{C}\right) \text {, }
$$

bzw. in Betriebsstoffwechsel $(=$ veratmet $)$

$$
C_{E}=(1-Y)\left(s_{R C}-s_{C}\right) \text {. }
$$

Nun ist aber zu präzisieren, dass von dem in den Bau- und Reservestoffwechsel eingehenden Substrat gewisse Mengen permanent festgelegt werden (z. B. in Zellwandmaterial, Kapseln usw.), deren relativer Anteil mit $Y_{\mathrm{S}}$ bezeichnet sei, und eine weitere Fraktion $Y_{T}$ als transitorische Reserve-

3) Die Umrechnung für $Q o_{e}$ von der Atmung bei der Messtemperatur $t_{e x p}$. zur Atmung bei der Temperatur $t$ im Belüftungsbecken erfolgt nach der Beziehung

bzw. bei $\Theta_{10}=2,0$

$$
Q_{O e\left(l^{\circ}\right)}=Q_{O e}\left(t_{\text {exp. }}\right) \quad \frac{1}{\operatorname{Num} \frac{\log \theta_{10}}{10}\left(t_{\text {exp. }}-t\right)} .
$$

$$
Q_{O e\left(t^{\circ}\right)}=Q_{O e\left(t_{e x p .)}\right.} \frac{1}{\operatorname{Num} \cdot\left[0,0301\left(t_{e x p}-t\right)\right]}
$$


stoffe fixiert werden, die beispielsweise zum Unterhalt der endogenen Atmung dienen. Die Fraktion $Y$ ist deshalb als Summe

$$
Y=Y_{S}+Y_{T}
$$

zu schreiben, und die als Überschußschlamm messbare, investierte Substratmenge wird dann

$$
\text { Überschußschlamm (gemessen })=Y_{S}\left(s_{R C}-s_{C}\right) \text {. }
$$

Die effektiv für die Substratatmung zu berïcksichtigende Menge eliminierten Kohlenstoffs wird dann

$$
\left(1-Y_{S}\right)\left(s_{R C}-s_{C}\right) \text {, }
$$

bzw. das mit der endogenen Atmung verbrauchte Substrat ist dann

$$
-Y_{T}\left(s_{R C}-s_{C}\right) \text {. }
$$

Diese letzte Fraktion kann nicht direkt bestimmt werden, hingegen kennen wir ihren Sauerstoffverbrauch aus der experimentellen Messung (vgl. vorhergehenden Abschnitt).

Wir dürfen damit rechnen, dass die gesamte bei der Substratatmung verbrauchte Menge von organischem $\mathrm{C}$ in Form von $\mathrm{CO}_{2}$ ausgeschieden wird. Nun wissen wir allerdings nicht, welche Sauerstoffmenge bei der Atmung benötigt wurde (der Sauerstoff im ausgeschiedenen $\mathrm{CO}_{2}$ stammt nicht aus der Luft; aller bei der Atmung verbrauchte Luftsauerstoff tritt ausschliesslich in Form von Wasser als Oxydationsendprodukt von Wasserstoff auf). Wir sollten deshalb die Respirationskoeffizienten $r_{q}=Q_{\mathrm{Co}_{2}} / Q_{\mathrm{O}_{2}}$ von Belebtschlamm, bzw. Belebtschlamm in Kontakt mit dem Abwasser kennen. Wenn $r_{q}$ angegeben werden kann, ergibt sich dann der Substrat-O-Verbrauch sofort zu

$$
Q_{O_{s}}=\left(1-Y_{S}\right)\left(s_{R C}-s_{C}\right) \frac{1}{r_{q}} \mathrm{~mol} \mathrm{O} / \mathrm{mol} \mathrm{C} .
$$

$\mathrm{Da}$ in der Technik normalerweise mit Gewichtseinheiten Sauerstoff gerechnet wird, können wir auch schreiben:

$$
Q_{O_{s}}=2,66 \frac{1}{r_{q}}\left(1-Y_{S}\right)\left(s_{R C}-s_{C}\right) \mathrm{g} \mathrm{O} / \mathrm{g} \mathrm{C} \text {. }
$$

Misst man $\left(s_{R}-s\right)$ als Abbauleistung $\left(s_{R}-s\right) \frac{R_{h}}{24}$ in $g$ organischem $\mathrm{C} / \mathrm{m}^{3}{ }_{B B} \cdot \mathrm{h}$, so erhält man $q_{s}$

$$
q_{s}=0,11 \frac{1}{r_{a}} R_{h}\left(1-Y_{S}\right)\left(s_{R C}-s_{C}\right) \quad\left[\mathrm{g} \mathrm{O} / \mathrm{m}^{\mathbf{3}}{ }_{B B} \cdot \mathrm{h}\right] \text {. }
$$

Für numerische Werte für $Y_{S}$ verweisen wir auf unsere frühere Arbeit (WUHRMANN [2I]). 


\section{Respirationskoeffizienten von Belebtschlamm}

Leider bestehen wenig Messungen über den Respirationskoeffizienten für die endogene und Substratatmung von Belebtschlamm. Tab. 6 und 7 enthalten einige Daten aus unseren eigenen Beobachtungen. Leider haben wir es unterlassen, diese Bestimmung in das Programm unseres Faktorenversuchs aufzunehmen, so dass das vorliegende Material etwas dürftig ist. Der Spielraum für $r_{q}$ ist aber an sich ziemlich gering, so dass Abschätzungen gemäss Formel (6a) für die Praxis doch mit ausreichender Genauigkeit vorgenommen werden können.

Tabelle 6

Respirationskoeffizienten von Belebtschlämmen*)

\begin{tabular}{llr}
\hline Belebtschlamm & Substrat & $v_{q}$ \\
\hline Häusliches Abwasser & & 0,75 \\
Häusliches Abwasser & endogen & 0,65 \\
Häusliches Abwasser & endogen & 0,66 \\
Häusliches Abwasser & endogen & 0,69 \\
Häusliches Abwasser & endogen & 0,71 \\
Häusliches Abwasser & endogen & 1,00 \\
Häusliches Abwasser & Glukose & 1,00 \\
Häusliches Abwasser & Saccharose & 0,66 \\
Häusliches Abwasser & Azetat & 0,43 \\
Häusliches Abwasser & Butyrat & 0,75 \\
Häusliches Abwasser & Aspartat & 0,80 \\
& Tryptophan & \\
\hline
\end{tabular}

*) Aus Wuhrmann und v. Beust [19].

Rechnet man mit einem Mittelwert von $r_{q}=0,65$ für die Veratmung von häuslichem Abwasser, so wird man keinen allzu grossen Fehler begehen.

\section{Substratatmung für die Nitrifizierung von Ammonium}

Der Sauerstoffverbrauch für die Veratmung des Ammoniums (sog. Nitrifizierung) in einer Belebtschlammanlage ist eine dominante Komponente des Substrat-O-Verbrauchs, die allerdings meistens unberücksichtigt bleibt. Dies ist ein grundsätzlicher Fehler, da in einem Belebtschlammsystem die Nitrifikation (d.h. das Wachstum der autotrophen nitrifizierenden Bakterien) nicht verhindert werden kann.

Der O-Verbrauch für die Oxydation von $\mathrm{NH}_{4}^{+}$zu $\mathrm{NO}_{2}^{-}$und $\mathrm{NO}_{3}^{-}$lässt sich annähernd quantitativ berechnen aus der Umsetzung $\mathrm{NH}_{4}^{+} \rightarrow \mathrm{NO}_{3}^{-}$ über $\mathrm{NO}_{2}^{-}$Der Einfachheit halber kann man die gesamte Abnahme $\left(s_{R_{N}}-s_{N}\right)$ 
Tabelle 7

Respirationskoeffizienten von Belebtschlämmen aus verschieden stark belasteten Anlagen

\begin{tabular}{|c|c|c|c|c|c|c|c|c|c|}
\hline Probe Nr. & $\begin{array}{l}\text { Betriebsdate } \\
R_{h} \\
\mathrm{~m}^{3} / \mathrm{m}^{3} \cdot \text { Tag }\end{array}$ & $\begin{array}{l}\text {, Belüftungsbecke } \\
R_{b} \\
\mathrm{~kg} \mathrm{BSB} / \mathrm{m}^{3} \cdot \mathrm{Tag}\end{array}$ & $\begin{array}{l}x_{1} \\
\mathrm{~kg} T S / \mathrm{m}^{3}\end{array}$ & $\begin{array}{l}S_{b} \\
\mathrm{~kg} \text { BSB } / \mathrm{kg} \cdot \mathrm{TS}\end{array}$ & $\begin{array}{l}\mathrm{O}_{2 B B} \\
\mathrm{mg} / \mathrm{l}\end{array}$ & $\begin{array}{l}t_{B B} \\
{ }^{\circ} \mathrm{C}\end{array}$ & $\begin{array}{l}\left.Q 0 e^{1}\right) \\
\mathrm{mg} \mathrm{O} / \mathrm{g} \mathrm{TS} \cdot \mathrm{h}\end{array}$ & $\begin{array}{r}r_{q}= \\
\text { endog. }\end{array}$ & $\begin{array}{l}\frac{\mathrm{CO}_{2}}{\mathrm{O}_{2}} \frac{\mu 1}{\mu 1} \\
\left.\quad \text { mit } R W^{3}\right)\end{array}$ \\
\hline 1 & 90 & $10 ; 8$ & 2280 & 4,72 & 4 & 15 & 16,7 & 0,62 & 0,62 \\
\hline $\begin{array}{l}1 \\
2\end{array}$ & 60 & 7,2 & 2600 & 2,77 & 4 & 15 & 16,5 & 0,63 & 0,68 \\
\hline 4 & 10,6 & 1,3 & 6950 & 0,19 & 3,5 & 13,4 & 4,05 & 0,63 & 0,58 \\
\hline 5 & 10,6 & 1,3 & 7080 & 0,18 & 3,5 & 16,4 & 3,74 & 0,55 & 0,43 \\
\hline 6 & 10,6 & 1,3 & 7100 & 0,18 & 3 & 16,8 & 4,72 & 0,63 & 0,61 \\
\hline 7 & 1 & 0,12 & 6570 & 0,018 & 6 & 16 & 1,62 & 0,60 & 0,65 \\
\hline 8 & 1 & 0,12 & 7500 & 0,016 & 6 & 16 & 1,98 & 0,61 & 0,65 \\
\hline 9 & 1 & 0,12 & 7740 & 0,016 & 6 & 16 & 2,30 & 0,56 & 0,69 \\
\hline & & & & & & & & 0,61 & 0,63 \\
\hline
\end{tabular}

$Q_{0}$ : endogene Respiration des gewaschenen, in Puffer resuspendierten Belebtschlamms bei $t=25^{\circ} \mathrm{C}$.

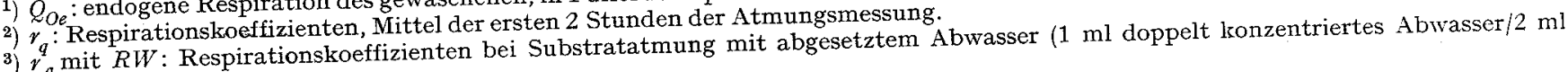

Schlammsuspension). 
der Ammoniumkonzentration in der Anlage für die Rechnung zugrunde legen, weil die in den Belebtschlamm inkorporierte $\mathrm{NH}_{4}^{+}$-Menge vernachlässigt werden kann. Pro Gramm nitrifizierten $\mathrm{NH}_{4}^{+}-\mathrm{N}$ werden $64 / 14=$ $4,6 \mathrm{~g} O$ benötigt. Man erhält dann

$$
\begin{aligned}
& Q_{o_{N}}=4,6\left(s_{R_{N}}-s_{N}\right) \quad\left[\mathrm{g} \mathrm{O} / \mathrm{m}^{3}\right] . \\
& \quad\left[\mathrm{g} \mathrm{O} / \mathrm{m}^{3}{ }_{B B} \cdot \mathrm{h}\right] .
\end{aligned}
$$

bzw.

$q_{N}=0,19 R_{h}\left(s_{R_{N}}-s_{N}\right)$

$\left(s_{R_{N}}-s_{N}\right)$ ist als g N/m ${ }_{B B}^{3} \cdot$ Tag zu messen.

So einfach die Berechnung des Sauerstoffbedarfs für die Nitrifikation ist, so schwierig gestaltet sich hingegen die Prognose, ob und wie gross in einem Belebtschlammsystem bei einer bestimmten, vorgeplanten Betriebsweise die Ammoniumoxydation ausfallen wird. $\mathrm{Zu}$ diesem Punkt sind folgende grundsätzliche Bemerkungen anzubringen:

a) Die Nitrifikation in einer Belebtschlammanlage tritt zwangsläufig ein, wenn bestimmte Voraussetzungen erf üllt sind. Es besteht dabei eine unausweichliche Wechselwirkung zwischen den Betriebsbedingungen, die zur Erzielung eines hohen Reinigungseffektes bezüglich der organischen Verunreinigungen notwendig sind, und den Bedingungen, bei denen die Nitrifikation auftritt.

b) DownING und HoPwoon [6] zeigten, dass die Gleichgewichtskonzentration von Nitrosomonas in einer Belebtschlammanlage proportional zum Verhältnis zwischen Schlammzuwachs und Schlammkonzentration im Belüftungsbecken ist (Formel 4 in [6]). Im Beharrungszustand ist diese Proportion gleichbedeutend mit dem Verhältnis von Schlammkonzentration und Überschußschlammenge $x_{1} / R_{h} x_{2}$, d.h. dem Schlammalter. Abb. 7 zeigt die Korrelation zwischen $x_{1} / R_{h} x_{2}$ und der prozentualen Verminderung der Ammoniumkonzentration (im wesentlichen durch Nitrifikation verursacht) bei den 27 Experimenten mit Sommertemperaturen unseres Faktorenversuchs. Man erkennt aus der Darstellung sofort, dass die Nitrifikation bei einem Verhältnis $x_{1} / R_{h} x_{2}^{2}$ von etwa $\mathrm{I}, 25$ plötzlich in Erscheinung tritt und dann übergangslos fast quantitativ erfolgt. Unter Berücksichtigung der Abwasserkonzentration (im Mittel etwa Io $\mathrm{mg} / \mathrm{NH}_{4}^{+}-\mathrm{N}$ ) und der Rücklaufschlammproportion von etwa I,2 ergibt sich eine sehr gute Übereinstimmung mit den theoretischen Berechnungen von DownING. In zweien unserer Experimente wurde trotz Erfüllung der theoretischen Voraussetzungen keine Nitrifikation beobachtet (in Abb. 7 in Klammer gesetzte Punkte), und zwar einfach deshalb, weil bei der relativ kurzen Dauer 


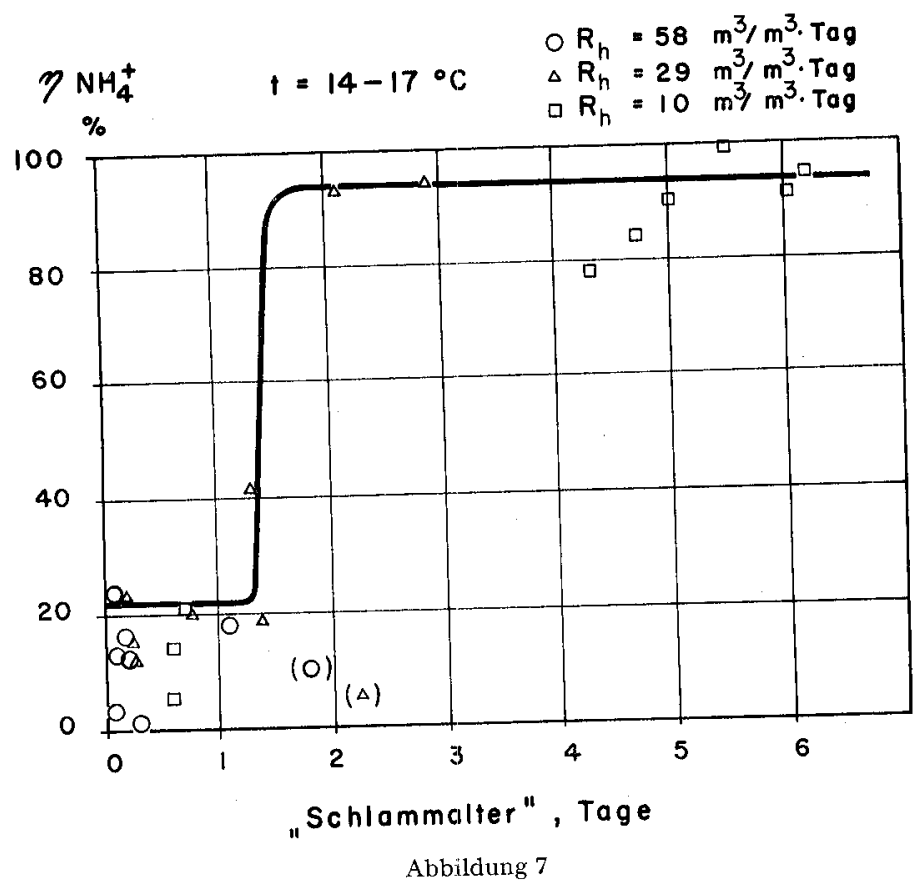

Prozentuale Verminderung des Ammoniumgehaltes im Abwasser in der biologischen Reinigung in Funktion des Verhältnisses $x_{1} / R_{h} x_{2}$ (Schlammalter) bei den Faktorenversuchen mit Temperaturen von $14-17^{\circ} \mathrm{C}$.

der einzelnen Versuche noch kein Beharrungszustand bezüglich der Populationsentwicklung $\mathrm{f}$ ür die nitrifizierenden Bakterien erreicht wurde.

c) Ein Einfluss der Sauerstoffspannung im Belüftungsbecken auf die Nitrifikation war in unseren Experimenten nur in geringem Ausmasse zu beobachten. Die Oxydation des Ammoniums kann also offensichtlich nicht mit der Sauerstoffspanung gesteuert werden, wenigstens nicht innerhalb jenes Bereiches, der für die normale oxydative Abwasserreinigung eingehalten werden muss.

d) Untersucht man die Nitrifikation in Funktion der Schlammbelastung (vgl. Abb. I in WuHrmann [22]), so ergibt sich, dass bei Temperaturen von $>13^{\circ} \mathrm{C}$ die Nitrifikation bei dem von uns untersuchten, verdünnten Abwasser unweigerlich eintritt, sobald Schlammbelastungen (gemessen am $\mathrm{BSB}_{5}$ ) von $S_{b}<\mathrm{I}$ (bei nur I mg O/l im Belüftungsbecken bei $S_{b}<0,7$ ) eingehalten werden. Dies sind aber gerade jene Grössenordnungen, die zur Erzielung eines gutgereinigten Abwassers (Vollreinigung) nicht überschritten werden sollten. Man darf sich bei der Beurteilung der Nitrifikation in 
einer Belebtschlammanlage nicht dadurch täuschen lassen, dass kein oder nur Spuren von Nitrat oder Nitrit im Abfluss nachgewiesen werden können. Die mit der Nitrifikation simultan immer mitgehende Denitrifikation kann in ihrer Geschwindigkeit völlig ausreichend sein, um eine ziemlich intensive Nitrifikation durch Reduktion des gebildeten Nitrats oder Nitrits gänzlich zu verdecken. Ein sicheres Urteil iiber Nitrifikation ist u.E. nur bei Betrachtung der $\mathrm{NH}_{4}^{+}$-Bilanz möglich, wobei eine gewisse Marge für den normalen Umsatz von Ammonium (Produktion und Assimilation) beim Stoffwechsel des Belebtschlamms eingerechnet werden muss (vgl. beispielsweise den Anfangsast der Kurve in Abb. 7).

Aus diesen Ausf ührungen ist zusammenfassend zu schliessen, dass bei niedrigen Schlammbelastungen, wie sie heute wobl zunebmend gefordert merden müssen, die Nitrifikation unvermeidlich ist und deshalb bei der Berechnung des Sauerstoffbedarfs unbedingt berïcksichtigt merden muss. Würde man diesem Bedarf keine Rechnung tragen, so ergäbe sich zwangsläufig die Tatsache, dass wegen mangelnder Leistung der Belüftungseinrichtung die Schlammkonzentration nicht bis zu jener Höhe gesteigert werden könnte, welche zur Erzielung des geforderten Reinigungsgrades eingehalten werden müsste.

\section{Berechnung des notwendigen Sauerstoffeintrags}

Aus der Beziehung ( $\mathrm{I}$ a) für das von der Belüftungseinrichtung zu verlangende $\mathrm{O}$-Eintragsvermögen $W_{c}$ bei der gewünschten $\mathrm{O}$-Konzentration $c$ im Belüftungsbecken erhält man nun als Summe aus den einzelnen Komponenten für den O-Bedarf:

$$
W_{c}=\frac{c_{s}}{c_{s}-c}\left(q_{e}+q_{s}+q_{N}\right)
$$

oder

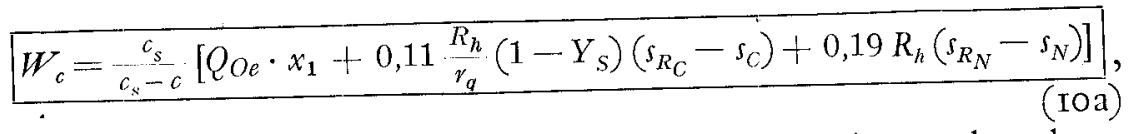

wobei $Q_{o e}$ und $c_{s}$ für die Temperatur im Belüftungsbecken zu berechnen sind.

Diese Berechnungsmethode ist streng kausal. Sie benötigt allerdings Masszahlen, die bisher bei der Abwasserreinigung nicht berücksichtigt wurden, insbesondere was die Abbauleistung für organischen $\mathrm{C}$ anbetrifft. Wir sind aber der Auffassung, dass auf andere Weise keine auch nur annähernd zuverlässige Abschätzung des $\mathrm{O}$-Eintrages möglich ist. Leider muss nochmals wiederholt werden, dass mit $\mathrm{BSB}_{5}$-Werten nicht operiert werden 
kann, weil eine Angabe für die entscheidenden Faktoren $Y_{S}$, bzw. $r_{q}$ auf der Basis des $\mathrm{BSB}_{5}$ nicht $\mathrm{zu}$ finden ist4).

Die Berechnung von $W_{c}$ nach Beziehung (Ioa) lässt sich für vollständig durchmischte Belüftungsbecken direkt anwenden. Bei längsdurchströmten Becken, in denen sowohl ein Gradient für die C-Substrate als auch für $\mathrm{NH}_{4}^{+}$ (bei Nitrifikation) auftritt, ist dieser Tatsache Rechnung zu tragen (stufenweise Berechnung). Man darf dabei annehmen, dass der Summand für die endogene Atmung im Längsprofil unverändert bleibt. Für seine Abschätzung darf man auch ohne weiteres die durchschnittliche Schlammbelastung ansetzen.

Beispiel: (durchmischtes Belüftungsbecken) Raumbelastung der Anlage Temperatur im Belüftungsbecken $\mathrm{O}_{2}$ im Belüftungsbecken

Rohwasser mit

\section{Belebtschlammkonzentration $x_{1}$}

Reinigungseffekt am organischen $\mathrm{C} \eta_{\mathrm{C}}$

$\mathrm{NH}_{4}^{+}$im Abfluss

$$
\mathrm{BSB}_{5} \eta_{b}
$$

$s_{R_{C}}-s_{C}$

$s_{R_{b}}-s_{b}$

$s_{R_{N}}-s_{N}$

$Q_{O e}$ bei $15{ }^{\circ} \mathrm{C}$

$Y_{S}$

$c_{s}\left(15^{\circ} \mathrm{C}, p=730 \mathrm{~mm}\right)$

$10 \mathrm{~m}^{3} / \mathrm{m}^{3} \cdot \operatorname{Tag}$

$15^{\circ} \mathrm{C}$

$2 \mathrm{mg} / \mathrm{l}$

$80 \mathrm{mg} \mathrm{C} / 1$

$20 \mathrm{mg} \mathrm{NH}_{4}-\mathrm{N} / \mathrm{l}$

$\mathrm{I} 20 \mathrm{mg} \mathrm{BSB} / 1$

$3000 \mathrm{mg} / \mathrm{l}$

$80 \%$

$90 \%$

Io $\mathrm{mg} \mathrm{NH}_{4}^{+}-\mathrm{N} / \mathrm{l}$

$64 \mathrm{mg} \mathrm{C/l}$

$108 \mathrm{mg} \mathrm{BSB} / 1$

Io $\mathrm{mg} \mathrm{N} / 1$

$0,5 Q_{O e}$ bei $25^{\circ} \mathrm{C}=6, \mathrm{og} \mathrm{O} / \mathrm{kg} \mathrm{TS} \cdot \mathrm{h}$

0,65

9,94

0,65

$r_{q}$

$$
\begin{aligned}
W_{C} & =-\frac{9994}{9,94-2}\left[6,0 \cdot 3+0, \mathrm{II} \cdot \frac{1}{0,65}(\mathrm{I}-0,65) 64 \cdot \mathrm{Io}+\mathrm{0,19} \cdot \mathrm{Io} \cdot \mathrm{IO}\right] \\
& =\mathrm{I}, 25(\mathrm{I} 8+37,9+\mathrm{I} 9) . \\
W_{c} & =93,6 \mathrm{~g} \mathrm{O} / \mathrm{m}^{3}{ }_{B B} \cdot \mathrm{h} .
\end{aligned}
$$

Ohne Nitrifikation ergibt sich $W_{c}=70 \mathrm{~g} \mathrm{O} / \mathrm{m}^{3}{ }_{B B} \cdot \mathrm{h}$, woraus sofort ersichtlich ist, dass die Nitrifikation von nur der Hälfte des $\mathrm{NH}_{4}^{++}$im Rohwasser den für die Oxydation der organischen Verunreinigungen benötigten O-Eintrag erwartungsgemäss beachtlich erhöht. Ferner ist nicht $\mathrm{zu}$

4) Es ist natürlich möglich, durch Gegenüberstellung von beispielsweise der $\mathrm{BSB}_{5}$-Belastung ner Anlage und "bewährten" Grössen des Sauerstoffeintrags auf rein empirischem Wege zu Bemessungszahlen zu kommen, welche einfach zu ermitteln sind. Anderseits zeigt die Praxis aber laufend, dass die Belïftungsleistung bei Belebtschlammanlagen fast immer ungenügend ist, sogar oft schon in den ersten Betriebsjahren, wenn die Belastungen noch weit vom Dimensionierungswert entfernt sind. Wir schliessen daraus, dass die angewendeten, rein empirischen Bemessungsregeln offenbar doch viel zu knappe Werte ergeben. 
übersehen, dass die Komponente der endogenen Atmung des Schlamms einen grossen Einfluss ausibt und keineswegs so gering eingeschätzt werden darf wie dies gelegentlich geschieht.

Führt man die Berechnung nach dem Vorschlag von VON DER EMDE [7] (S. II3) mit der Beziehung (wir wählen unsere Bezeichnungen)

$$
W_{c}=0,5\left(s_{R_{b}}-s_{b}\right) R_{h}+0,1 x_{1}
$$

durch, so erhält man

$$
\begin{gathered}
W_{c}=0,5 \cdot 0,108 \cdot \mathrm{Io}+0, \mathrm{I} \cdot 3 \mathrm{~kg} \mathrm{O} / \mathrm{m}^{3} \cdot \mathrm{Tag}=0,80 \mathrm{~kg} \mathrm{O} / \mathrm{m}^{3}{ }_{B B} \cdot \mathrm{Tag}, \\
\mathrm{bzw} \cdot=33,3 \mathrm{~g} \mathrm{O} / \mathrm{m}^{3}{ }_{B B} \cdot \mathrm{h},
\end{gathered}
$$

was für die angegebenen Bedingungen des Anlagenbetriebes gemäss unseren experimentellen Beobachtungen (sogar bei Vernachlässigung der Nitrifikation) viel zuwenig ist.

\section{Summary}

Basic Factors for the Dimensioning of Aeration in Actipated Sludge Plants

The generally accepted theory for the calculation of the oxygen consumption caused by the microbes in an aeration basin assumes that the total of oxygen utilized $q_{g}$ is the sum of the endogenous respiration of the sludge $q_{e}$ plus a quantity for substrate respiration $q_{s}$. This theory - though physiologically probably not quite correct -, is very convenient because it allows a causal calculation of the necessary oxygen input into an activated sludge plant.

Extensive data on endogenous respiration of activated sludge are presented (Table $\mathrm{I}$ and 5 ) and are correlated with sludge load and sludge age (Fig. 4 and 5).

The term substrate respiration is discussed and a calculation method, based on the removal of organic $\mathrm{C}$ by the mixed liquor, is proposed. In view of the fact that nitrification is an inevitable reaction in activated sludge treatment (its extent being strictly a function of the operation parameters of a plant), oxygen consumption for the oxidation of ammonia has to be included into the term of substrate respiration. The amount of this oxygen fraction has to be predicted from the degree of nitrification to be expected at the desired operation conditions.

The following formula for the calculation of the oxygen input into a completely mixed aeration basin is proposed (explanations of symbols see annex):

$$
W_{c}=\frac{c_{s}}{c_{s}-c}\left(q_{e}+q_{S}+q_{N}\right)
$$




$$
\begin{aligned}
W_{c}=\frac{c_{8}}{c_{\mathrm{s}}-c}[ & Q_{O e} \cdot x_{1}+0, \mathrm{II} \frac{R_{h}}{v_{q}}\left(\mathrm{I}-Y_{S}\right)\left(s_{R_{C}}-s_{C}\right) \\
& \left.+0, \mathrm{Ig} R_{h}\left(s_{R_{N}}-s_{N}\right)\right] \quad\left[\mathrm{gO} / \mathrm{m}^{3}{ }_{A B} \cdot \mathrm{h}\right] .
\end{aligned}
$$

This same calculation method may be applied stepwise for aeration basins of the tubular reactor type (longitudinal flow basins) considering the concentration decrease of the substrates along the length profile of the basin (the term for endogenous respiration can be assumed constant for the entire aeration time).

\section{Bezeichnungen (Symbols):}

$c_{s} \quad$ Sättigungskonzentration des Belüftungsbeckeninhaltes mit Sauerstoff bei $t^{\circ}$ und örtlichem mittlerem Luftdruck $p$ ig $\mathrm{O} / \mathrm{m}^{3}$ ]

Oxygen saturation concentration of mixed liquor at $t^{\circ}$ and average local barometric pressure $p \quad\left[\mathrm{~g} \mathrm{O} / \mathrm{m}^{3}\right]$

Gewünschte Sauerstoffkonzentration im Belüftungsbecken bei Beharrungszustand $\left[\mathrm{g} \mathrm{O} / \mathrm{m}^{3}\right]$

Desired oxygen concentration in the mixed liquor at steady state conditions

$k_{\mathrm{t}} \quad$ Belüftungskonstante der Belüftungseinrichtung für das Schlamm-Abwassergemisch im Belüftungsbecken bei $t^{\circ} \quad\left[\mathrm{h}^{-1}\right)$

Oxygenation constant of the aeration mechanism for mixed liquor at $t^{\circ}$

$W_{\mathrm{o}}$ Sauerstoffeintragsvermögen der Belüftungseinrichtung bei $c=0 \mathrm{mg} \mathrm{O} / 1, t^{\circ}$ und mittlerem örtlichem Luftdruck $\not{p} \quad\left[\mathrm{~g} \mathrm{O}^{3} \mathrm{~m}^{3}{ }_{B B} \cdot \mathrm{h}\right]$

Oxygenation capacity of the aeration basin at $c=0 \mathrm{mg} O / 1, t^{\circ}$ and average local barometric pressure $p$ [g $\left.\mathrm{O} / \mathrm{m}^{3} A B \cdot \mathrm{h}\right]$

$W_{c}{ }_{c}$ Sauerstoffeintragsvermögen bei $c \mathrm{mg} \mathrm{O} / \mathrm{l}$ im Belüftungsbecken $\left[\mathrm{g} \mathrm{O} / \mathrm{m}^{3}{ }_{B B} \cdot \mathrm{h}\right]$

Oxygenation capacity at $c \mathrm{mg} \mathrm{O} / \mathrm{l}$ in the mixed liquor $\left[\mathrm{g} \mathrm{O} / \mathrm{m}^{3} A B \cdot \mathrm{h}\right.$ ]
Endogene Atmung des Belebtschlamms [g O/kg Trockensubstanz $\cdot \mathrm{h}$ ]

Endogenous respiration of activated sludge $[\mathrm{g} \mathrm{O} / \mathrm{kg}$ dry solids $\cdot \mathrm{h}$ ]

$Q_{O s} \quad$ Substratatmung des Belebtschlamms $\left[\mathrm{g} \mathrm{O} / \mathrm{m}^{3}\right]$

Substrate respiration of activated sludge $\left[\mathrm{g} \mathrm{O} / \mathrm{m}^{3}\right]$

$Q_{O}$ Substratatmung des Belebtschlamms für die Nitrifikation von $\mathrm{NH}_{4}^{+} \cdot\left[\mathrm{g} \mathrm{O} / \mathrm{m}^{3}\right]$

Substrate respiration for nitrification $\left[\mathrm{g} \mathrm{O}^{3} \mathrm{~m}^{3}\right]$

$q_{e}$

Endogene Atmung des Schlamms $\left[\mathrm{g} O / \mathrm{m}^{3} B B \cdot \mathrm{h}\right]$

Endogenous respiration of mixed licuor $\left[\mathrm{g} \mathrm{O} / \mathrm{m}^{3} A B \cdot \mathrm{h}\right]$

$q_{s} \quad$ Substratatmung des Schlamms $\left[\mathrm{g} \mathrm{O} / \mathrm{m}^{3}{ }_{B B} \cdot \mathrm{h}\right]$

$q_{s} \quad$ Substrate respiration of mixed liquor $\left[\mathrm{gO} / \mathrm{m}^{3} A B \cdot \mathrm{h}\right]$

$q_{N} \quad$ Substratatmung für Nitrifikation $\left[\mathrm{g} \mathrm{O}^{\circ} / \mathrm{m}_{B B}^{3} \cdot \mathrm{h}\right]$

Substrate respiration for nitrification $\left[\mathrm{g} \mathrm{O} / \mathrm{m}^{3} A B \cdot \mathrm{h}\right]$

$q_{g}$

Gesamter Sauerstoffverbrauch im Belüftungsbecken $\left[\mathrm{g} \mathrm{O} / \mathrm{m}_{B B}{ }_{B B} \mathrm{~h}\right]$

Total oxygen consumption by the mixed liquor $\left[\mathrm{g} \mathrm{O}^{\mathrm{O}} / \mathrm{m}^{3} A B \cdot \mathrm{h}\right]$

$r_{q} \quad$ Respirationskoeffizient $\left[\mathrm{molCO}_{2} / \mathrm{molO}_{2}\right]$

Respiration quotient $\left[\mathrm{molCO} / \mathrm{molO}_{2}\right]$
$s_{R} \quad$ Substratkonzentration im Belüftungsbeckenzufluss $\left[\mathrm{g} / \mathrm{m}^{3}\right]$

${ }^{s}{ }_{R} \quad$ Substrate concentration in aerator influent $\left[\mathrm{g} / \mathrm{m}^{3}\right]$

s Dito im Abfluss

do. aerator effluent

Indices C bzw. $\mathrm{N}$ bzw. $b$ beziehen sich auf Substrat als organischer $\mathrm{C}$ bzw. $\mathrm{NH}_{4}^{+}$ bzw. BSB

Indices $\mathrm{C}$ and $\mathrm{N}$ and $b$ stand for organic $\mathrm{C}, \mathrm{NH}_{\mathbf{4}}^{+}$and BOD respectively

$Y \quad$ Ausnützungsfaktor für das Substrat [g Schlammzuwachs/g eliminiertes Substrat] Yield factor of substrate utilization $[\mathrm{g}$ sludge weight increase/g substrate eliminated] 
$Y_{S} \quad$ Ausnützungsfaktor für das Substrat, soweit er zur Überschußschlammbildung führt [g Ưberschußschlamm/g eliminiertes Substrat]

Yield factor of substrate utilization for excess sludge production [g excess sludge/g substrate utilized]

$Y_{T}$ Ausnützungsfaktor für das Substrat bei der Bildung transitorischer Reservestoffe [g gebildete Reservestoffe/g eliminiertes Substrat]

Yield factor of substrate utilization for formation of transitory reserve material [g reserve material/g substrate utilized]

$R_{h} \quad$ Hydraulische Raumbelastung $\left[\mathrm{m}^{3}\right.$ Abwasser $/ \mathrm{m}^{3}$ Belüftungsraum. Tag]

Hydraulic load of aeration basin $\left[\mathrm{m}^{3} / \mathrm{m}^{3} A B \cdot d\right]$

$S_{b} \quad$ Schlammbelastung mit $\mathrm{BSB}_{5} \quad[\mathrm{~kg}$ BSB/kg Trockensubstanz $\cdot \mathrm{Tag}]$

Sludge load of mixed liquor [ $\mathrm{kg} B O D / \mathrm{kg}$ solids $\cdot \mathrm{d}]$

$x_{1} \quad$ Schlammkonzentration im Belüftungsbecken $\left[\mathrm{kg}\right.$ Trockensubstanz $\left./ \mathrm{m}^{3}\right]$

Solids concentration of mixed liquor $\left[\mathrm{kg} / \mathrm{m}^{3}\right]$

$x_{2}$ Uberschußschlamm [ $\mathrm{kg}$ Trockensubstanz $/ \mathrm{m}^{3}$ Abwasser]

Excess sludge [kg dry solids $/ \mathrm{m}^{3}$ sewage]

I Belïftungsbeckeninhalt $\left[\mathrm{m}^{3}\right]$

Volume of aeration basin $\left[\mathrm{m}^{3}\right]$

$B B$ Belüftungsbecken

$A B$ Aeration basin

\section{LITERATURVERZEICHNIS}

[I] Barker, H.A., The Oxydative Metabolism of the Colorless Alga, Prototbeka zopfit, J. Cellular Comp. Physiol. 8, I75-182 (1936).

[2] Clufton, C. E., Microbial Assimilations, Advances in Enzymol. 6, 269-308 (1946). [3] CLIFTON, C.E., and SOBEK, J. M., Oxidatipe Assimilation and Endogenous Respiration of Bacillus cereus., J. Bact. 81, 284-287 (1961).

[4] Clifton, C.E., and SobEk, J. M., Endogenus Respiration of Bacillus cereus., J. Bact. 81, $252-256$ (196I).

[5] Clifton, C.E., Oxydatide Assimilation and Distribution of Glucose in Bacillus cereus, J. Bact. $83,66-69$ (1962).

[6] Downing, A.L., and Hopwood, A.P., Some Observations on the Kinetics of Nitrifying Activated-Sludge Plants, Schweiz. Z. Hydrol. 26, (1964) 27I-288.

[7] VON DER EMDE, W., Beitrag zu Versucben zur Abmasserreinigung mit belebtem Schlamm. Veröffentl. d. Inst. f. Siedlungswasserwirtsch., TH Hannover, H. I (1957).

[8] HÖRLER, A., Lrgebnisse pon Versuchen der EAWAG mit dem «Kombibecken», Schweiz. 7. Hydrol. 26 (1964).

[9] HUNKEN, K.H., Untersuchungen über den Reinigungsperlauf und den Sauerstoffperbraucb bei der Abmasserreinigung durcb das Belebtschlammperfabren, Stúttgarter Ber. z. Siedlungswasserwirtschaft, Nr. 4 (R. Oldenburg, Verlag, München I960).

[10] Keefer, C.E., and Meisel, J.T., Activated Sludge Studies. IV. Sludge Age and its Iffect on the Activated Sludge Process, Sew. Ind. Wastes 25, 898-908 (1953).

[ i I] Orford, H. E., HeUkelekian, H., and Isenberg, E., Effect of Sludge Loading and Dissolved Oxygen on the Performance of the Activated Sludge Process, in: Advances in Biological Waste Treatment, Proc. 3rd Conf. on Biological Waste Treatment, Manhattan Coll. 1960, 25 I-263 (Pergamon Press, Oxford 1963).

[12] RAMSEY, H.H., Endogenous Respiration of Staphylococcus aureus, J. Bact. 83, 507-5I 4 (1962). 
[13] Ruchhoft, C. C., McNamee, P. D., and Butterfield, C.'T., Studies on Semage Purification. VII. Biochemical Oxydation by Activated Sludge, Sew. Wks. J. 10, 66I-690(1938).

[14] RuchHoFT, C.C., and Smith, R.S., Studies of Semage Purification. X. Changes in Characteristics of Activated Sludge Induced by Variations in Applied Load, Sew. Wks. J. 11, 409-425 (I939).

[15] VIEHL, K., Der Linfluss des Sanerstoffgebaltes auf die biologische Abmasserreinigung, Zbl. Bakt. II, 104, I6I (I94I/42).

[i6] WoOldridge, W.R., and StandFast, A.F.S., Some Experiments on the Oxydation of Sludge and Sludge Semage Systems, Biochem, J. 30, I49 (1936).

[I7] Wuhrmann, K., Factors Affecting Efficiency and Solids Production in the Activated Sludge Process, in: Biological Treatment of Sewage and Industrial Wastes, 49-65 (Reinhold Publ. Corp., New York 1956).

[I 8] WUHRMANN, K., Über die Sauerstoffersorgung flockiger oder zoogloenartiger Aggregationen aerober Mikroorganismen, Z. allg. Path. u. Bakt. 20, 567-576 (1957).

[19] WuHrmanN, K., und von Beust, F., Zur Theorie des Belebtschlammverfabrens. II. Über den Mechanismus der Elimination gelöster organischer Stoffe aus Abwasser bei der biologischen Reinigung, Schweiz. Z. Hydrol. 20, 3 I I-330 (I958).

[20] Wuhrmann, K., Effect of Oxygen Tension on Biocbemical Reactions in Semage Purification Processes, in: Advances in Biological Waste Treatment, Proc. 3 rd Conf. on Biol. Waste Treatment, Manhattan College I960, 27-40 (Pergamon Press, Oxford 1963).

[2I] WUHRMANN, K., Hauptwirkungen und Wechselwirkungen einiger Betriebsparameter im Belebtschlammsystem, Ergebnisse mebrjäbriger Grosswersuche, Schweiz. 'Z. Hydrol. 26, 21 8-270 (1964).

[22] Wuhrmann, K., Stickstoff- und Pbospborelimination, Ergebnisse von Versuchen im tecbniscben Maßstab, Schweiz. Z. Hydrol. 26, 520 (1964).

Separatdruck aus der Schweiz. Zeitschrift für Hydrologie 26, Fasc. 2 (1964) Herausgegeben mit Unterstüzung der Stiftung der Wirtschaft zur Förderung des Gewässerschutzes in der Schweiz 